Check for updates

Cite this: RSC Adv., 2018, 8, 39496

Received 4th September 2018

Accepted 19th November 2018

DOI: $10.1039 / \mathrm{c} 8 \mathrm{ra07377e}$

rsc.li/rsc-advances

\section{A modern and practical laccase-catalysed route suitable for the synthesis of 2-arylbenzimidazoles and 2-arylbenzothiazoles}

\author{
Mudzuli Maphupha, (D) Wanyama P. Juma, Charles B. de Koning and Dean Brady (D)* \\ Heterocyclic aromatic compounds containing an imine $(\mathrm{C}=\mathrm{N})$ bond such as benzimidazoles and \\ benzothiazoles are important active pharmaceutical ingredients. The synthesis of 2-aryl-1H- \\ benzimidazoles and 2-arylbenzothiazoles in good to excellent yields was achieved by reacting 2- \\ aminoaromatics with various benzaldehyde derivatives catalysed by the commercial laccases Novoprime \\ and Suberase ${ }^{\circledR}$ at room temperature and in the presence of atmospheric oxygen.
}

\section{Introduction}

Applying enzymes to address modern challenges experienced in complex synthetic organic chemistry has proven valuable. Enzymes provide alternative and sustainable processes and have helped to minimize the release of hazardous substances into the environment. ${ }^{1}$ Laccases (benzenediol : oxygen oxidoreductases, EC1.10.3.2) are well recognized oxidoreductase enzymes belonging to the family of blue multi-coppercontaining oxidases. ${ }^{1}$ They are capable of catalysing oxidation reactions of several low molecular weight organic compounds such as polyphenols, aminophenols, methoxyphenols, aminophenols, and lignin-related molecules. ${ }^{1-4}$ The catalytic process of these enzymes occurs through a one-electron oxidation and water is released as the by-product. ${ }^{1}$ Because of their broad catalytic activity, laccases have been utilised in a variety of industrial applications ranging from textile to the pulp and paper industries and from food industries to bioremediation processes. $^{5}$

Benzimidazoles are a class of heterocyclic aromatic compounds composed of an imidazole ring fused to a benzene ring. Because of their remarkable biological activities, benzimidazoles have been studied for more than a century and are a common moiety in many active pharmaceuticals (Fig. 1). One of the prominent natural occurring derivatives of benzimidazoles is $\mathrm{N}$-ribosyl-dimethyl benzimidazole; a component of inhibitors such as factor Xa (Fxa) inhibitors, poly (ADP-ribose) polymerase (PARP) inhibitors, 5-HT3 antagonists, and 5-lipoxygenase inhibitors, and also serves as one of the cobalt ligands in vitamin B12. ${ }^{6}$ Substituted benzimidazoles are associated with a wide range of biological and pharmacological activities such as anti-tumour, anti-ulcer, anti-fungal, antihypersensitive, antiviral agents, anti-allergic properties, as

Molecular Sciences Institute, School of Chemistry, University of the Witwatersrand, Johannesburg, South Africa. E-mail: dean.brady@wits.ac.za; Tel: +27-11-7176745 well as neuropeptide Y Y1 receptor antagonists. ${ }^{7,8}$ Furthermore, through blocking the secretion of gastric acid in the stomach, substituted benzimidazoles such as omeprazole can act as gastric $\mathrm{H}^{+} / \mathrm{K}^{+}$ATPase inhibitors. ${ }^{9-11}$ Some of the well-known available drugs bearing a benzimidazole scaffold include albendazole, mebendazole, omeprazole, and bendamustine (Fig. 1). ${ }^{12}$ Furthermore, compounds with benzimidazole moieties have been utilized in material science applications; they are often used as membranes for fuel cells and in organic lightemitting diodes. ${ }^{13}$

A related class of compounds, the benzothiazoles, are also an important class of aromatic heterocyclic compounds composed of a benzene ring fused to a thiazole ring where a thiazole ring is a five membered ring made up of sulphur and nitrogen atoms. ${ }^{\mathbf{1 4 , 1 5}}$ The benzothiazole moiety substituted with various functional groups has attracted attention due to their pharmacological and therapeutic activities (Fig. 2). ${ }^{\mathbf{1 4}}$ These compounds have displayed antitumor, ${ }^{16}$ anticancer, ${ }^{14,15,17}$ anti$\mathrm{HIV}^{18}$ antiviral, ${ }^{19}$ antimicrobial, ${ }^{20}$ antibacterial, ${ }^{21}$ anthelmintic, ${ }^{22}$ anti-diabetic, ${ }^{23}$ anti-allergic ${ }^{24}$ and anti-inflammatory activities. ${ }^{25}$ Other reported applications include use in industry as oxidants, industrial dyes and functional materials. ${ }^{16,26-33}$
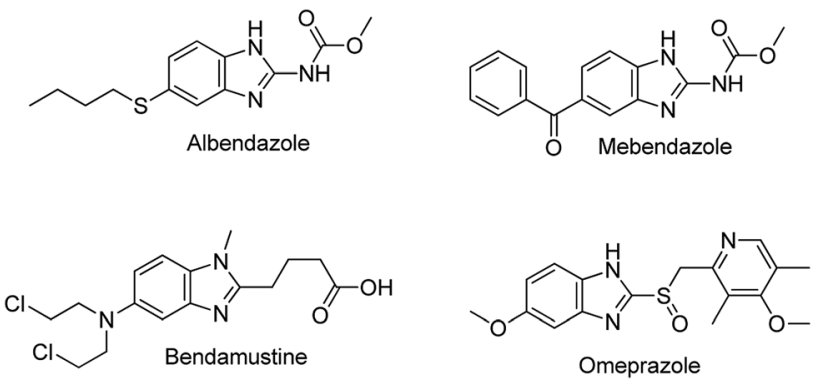

Fig. 1 Pharmaceuticals a benzimidazole nucleus. 


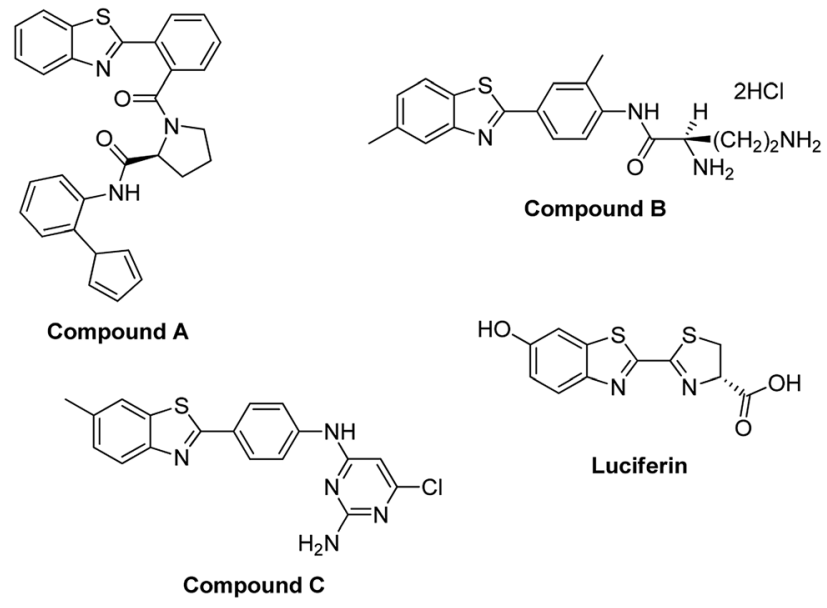

Fig. 2 Benzothiazoles in biologically active compounds.

Due to their numerous biological activities, development of efficient synthesis methods for benzimidazoles and benzothiazoles compounds has received a significant amount of interest. ${ }^{7,12,14}$ The conventional methods include condensationdehydration reaction of ortho-aminoaromatics with carboxylic acids and their derivatives under strong acid and high temperature conditions or condensation with aldehydes under oxidative conditions. ${ }^{26,34} \mathrm{~A}$ number of oxidants have been used to catalyse these reactions, including iodine, peroxides, 1,4benzoquinone, nitrobenzene, cupric acetate, and (bromodimethyl)sulfonium bromide, and recent advances for benzimidazoles include procedures such as microwave assisted synthesis and solid phase synthesis. ${ }^{10}$ The use of transitionmetal catalysed cross-couplings reactions of benzothiazoles and aromatic halides using aromatic boronic and carboxylic acids is also known. ${ }^{27}$

Most reported methods are disadvantageous because they involve use of toxic oxidants, abrasive reaction conditions (high temperatures and pressure, as well as long reaction times). Furthermore, the transition metals are expensive while solvents such as NMP, DCM, and DMF are environmentally unfriendly. ${ }^{26,27}$

Greener approaches have been reported recently; this includes the laccase catalysed reaction between $o$-phenylenediamine and benzaldehydes as well as the two-step laccasemediator aerobic oxidation condensation of in situ-produced salicylaldehyde derivatives with aromatic amines for the synthesis of benzimidazole derivatives. ${ }^{7}$

Therefore, alternative and more environmental friendly preparation of 2-aryl-1 $\mathrm{H}$-benzimidazoles and 2-arylbenzothiazoles involves enzymes. ${ }^{1,7}$ Herein, we report laccase catalysed one-pot syntheses of 2-aryl-1 $H$-benzimidazoles and 2arylbenzothiazoles from a condensation-dehydration reaction of 2-aminoaromatics with aryl-aldehydes.

\section{Results and discussion}

The synthesis of benzimidazole derivatives can be achieved through reactions of derivatives of $o$-phenylenediamine 1 with aldehydes 2 with using molecular oxygen as an oxidant. However, this results not only in the formation of 2substituted benzimidazole (3) but also 2,3-disubstituted benzimidazole (4) as a by-product or even the major product (Scheme 1). ${ }^{7}$ Hence, although this protocol was effective, poor chemoselectivity was a disadvantage. Recently, Leutbecher et al. discovered that the same reaction using the laccase catalysed enzyme domino reaction between $o$-phenylenediamine 1 with various aromatic aldehydes 2 in aerobic conditions using a phosphate buffer and obtained 2-aryl- $1 H$-benzimidazoles 3 in good to excellent yields but with some disubstituted by-product 4 . $^{7}$

It has been shown that in the presence of an oxidizing agent the reaction of $\mathbf{1}$ with $\mathbf{2}$ favours the formation of product $\mathbf{3}$ over product $4 .{ }^{34}$ Following this, our aim was to first identify how and why the 1- $N$-benzylated product $\mathbf{4}$ was formed and if its formation could be controlled and possibly eliminated. Our initial approach was to investigate the selectivity of these enzymefacilitated reactions by varying the $\mathrm{pH}$, types of buffers and co-solvents used in the synthesis of these compounds. The cosolvents used in this study were methanol, ethanol, DMF, and acetonitrile and the buffers were acetate and phosphate at various $\mathrm{pH}$ values.

As a starting point, we investigated the reaction between $o$ phenylenediamine (1a) with benzaldehydes 2a-d (Fig. 3) (1:2 ratio) in acetate buffer ( $\mathrm{pH} 4.5)$ using the laccase Suberase ${ }^{\circledR}$ from Novozymes at room temperature (Scheme 1). Initially, the reaction times for these reactions varied from 2 to $24 \mathrm{~h}$; it was observed that for the majority of the substrates, the monosubstituted product (3) forms first and then followed by the undesired $N$-benzylated product (4). Therefore, a reaction time of $24 \mathrm{~h}$ was selected for the model studies to detect the formation of by-product compound 4 .

Secondly, the relative ratios of products 3 and 4 obtained by laccase-catalysed oxidation were profoundly influenced by the type of organic solvent used. As has been observed previously inferior results were obtained while using methanol and ethanol as co-solvents, as they both showed a great deal of competitive formation of products 3 and 4.,12 Improved chemoselectivity was observed with DMF and acetonitrile; wherein we were able to minimize the formation of the $1-N$-benzylated product 4 (Table 1).

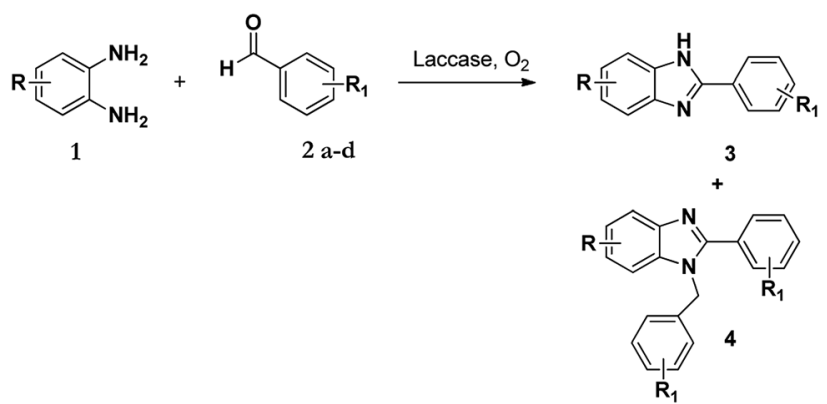

Scheme 1 Laccase catalysed reaction of 1 and 2 in acetate buffer $(\mathrm{pH}$ 4.5) using various co-solvents. 


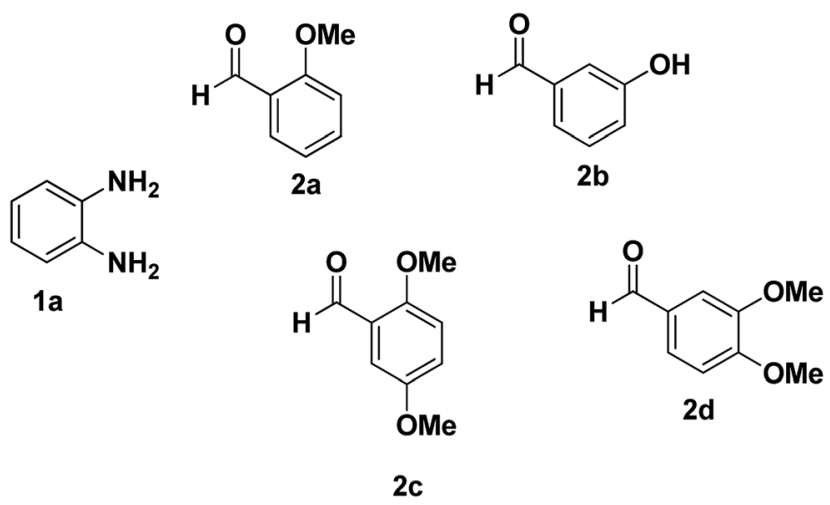

Fig. 3 o-Phenylenediamine (1a) and aromatic aldehydes ( $2 a-d)$ used in this study.

Thirdly, we explored the possible effect of the type of laccase used on selectivity of these reactions. When Fan et al. developed a highly chemoselective scandium(II) triflate based method for synthesis of either 2-benzimidazoles or 2,3-benzimidazoles it was demonstrated that the presence of a strong oxidizing agent such as hydrogen peroxide influences the production of the 2substituted benzimidazoles over the 2,3-disubstituted benzimidazoles. This implies that the redox potential of the laccase used could influence the chemoselectivity of the reactions. ${ }^{35,36}$

Therefore, using laccases (Suberase ${ }^{\circledR}$, Denilite $₫$ II Base, and Novoprime Base 268) from Novozymes, we investigated the role of enzyme type and preparation on these reactions. The reaction between $o$-phenylenediamine (1a) with benzaldehyde $\mathbf{2 d}$ was chosen as a model reaction and it was performed using an acetate buffer ( $\mathrm{pH}$ 4.5) and acetonitrile at room temperature (Scheme 1).

The findings shown in Fig. 4, clearly indicate that the specific laccase influences the chemoselectivity of benzimidazole derivatives to a great extent, based on the redox potential of

Table 1 Suberase ${ }^{\circledR}$-catalysed formation of benzimidazoles through reaction of 0 -phenylenediamine (1a) and benzaldehydes $2 a-d$ in acetate buffer (0.1 M, pH 4.0) using various co-solvents ${ }^{a}$

\begin{tabular}{llllll}
\hline Entry & Aldehyde & Co-solvent & Time & 3 Yield \% & 4 Yield \% \\
\hline 1 & $2 \mathrm{a}$ & Methanol & $24 \mathrm{~h}$ & 59 & 20 \\
2 & $2 \mathrm{a}$ & Ethanol & $24 \mathrm{~h}$ & - & 13 \\
3 & $2 \mathrm{a}$ & DMF & $24 \mathrm{~h}$ & 72 & - \\
4 & $2 \mathrm{a}$ & Acetonitrile & $24 \mathrm{~h}$ & 62 & - \\
5 & $2 \mathrm{~b}$ & Methanol & $24 \mathrm{~h}$ & - & - \\
6 & $2 \mathrm{~b}$ & Ethanol & $24 \mathrm{~h}$ & - & 97 \\
7 & $2 \mathrm{~b}$ & DMF & $24 \mathrm{~h}$ & - & 98 \\
8 & $2 \mathrm{~b}$ & Acetonitrile & $24 \mathrm{~h}$ & - & 82 \\
9 & $2 \mathrm{c}$ & Methanol & $24 \mathrm{~h}$ & 10 & 90 \\
10 & $2 \mathrm{c}$ & Ethanol & $24 \mathrm{~h}$ & 25 & 70 \\
11 & $2 \mathrm{c}$ & DMF & $24 \mathrm{~h}$ & - & 74 \\
12 & $2 \mathrm{c}$ & Acetonitrile & $24 \mathrm{~h}$ & - & 56 \\
13 & $2 \mathrm{~d}$ & Methanol & $24 \mathrm{~h}$ & 34 & 66 \\
14 & $2 \mathrm{~d}$ & Ethanol & $24 \mathrm{~h}$ & - & - \\
15 & $2 \mathrm{~d}$ & DMF & $24 \mathrm{~h}$ & 67 & 23 \\
16 & $2 \mathrm{~d}$ & Acetonitrile & $24 \mathrm{~h}$ & 59 & 36
\end{tabular}

${ }^{a}$ Reaction conditions: Myceliophthora thermophilia laccase $(2.0 \mathrm{~mL}$ Suberase $\left.{ }^{\circledR}\right) 50: 50$ solvent/acetate buffer at $25^{\circ} \mathrm{C}$. each enzyme. Novoprime Base 268 presented the best oxidation results compared to the two laccases preparations from $\mathrm{Myce}$ liophthora thermophile (Suberase ${ }^{\circledR}$ and Denilite ${ }^{\circledR}$ II Base) for the formation of 2-(3,4-dimethoxyphenyl)-1H-benzimidazole (3d). The reaction conducted using Suberase ${ }^{\circledR}$ as our laccase afforded a yield of $59 \%$ of the 2-substituted benzimidazole $3 \mathbf{d}$ and $36 \%$ was the 3,4-disubstituted benzimidazole $4 d$. When using Denilite ${ }^{\circledR}$ II Base as our laccase, the ratio was $57 \%$ compound 3d and 30\% compound 4d. Finally, when the enzyme Novoprime Base 268 was used, compound 3d was formed in a $78 \%$ yield while compound $\mathbf{4 d}$ was not detected under these reaction conditions.

Finally, in order to identify the optimal conditions for these reactions we also considered the effects of using different buffers and pH towards the reaction selectivity. Leutbecher et al. investigated the effect of different buffers using acetate and phosphate buffers while varying buffer to co-solvent ratios. It was shown that the ratios of buffer to co-solvent affect chemoselectivity and consequently the yield of the products formed.7 In this study the buffer and $\mathrm{pH}$ studies were conducted using phosphate buffer $(0.1 \mathrm{M}, \mathrm{pH} 6.0-7.5)$ and acetate buffer $(0.1 \mathrm{M}$, pH 3.0-5.0) with DMF or acetonitrile as co-solvents at room temperature. Reactions at lower $\mathrm{pH}$ values (3.0-5.0) occurred at faster rates than reactions carried out at higher $\mathrm{pH}$ values (>7.15), but no significant effect on selectivity was observed.

Using the discovered optimal conditions (General method), derivatives of $o$-phenylenediamine were treated with a series of aromatic aldehydes using commercial laccase, Novoprime Base 268, from Novozymes at room temperature and the obtained results are summarised in Table 2 below.

As shown in Table 2, 2-aryl-1 $H$-benzimidazoles were obtained in good to excellent yields (60-94\%) from a variety of aromatic aldehydes. It is clear the method applies for a variety of aryl aldehydes containing both electron-donating and electron-withdrawing substituents around the ring. No byproduct (4) was formed in any of the reactions. For compounds $\mathbf{3 e}, \mathbf{5 c}$ and $\mathbf{5 d}$ by-product was previously observed by Leutbecher et al. in ratios of $20: 3,2: 1$ and $10: 1$ respectively in the absence of laccase. ${ }^{7}$

As shown in Scheme 2 below; after the condensation of the diamine and the aromatic aldehyde, the subsequent intermediate benzimidazoline i may condense further with another

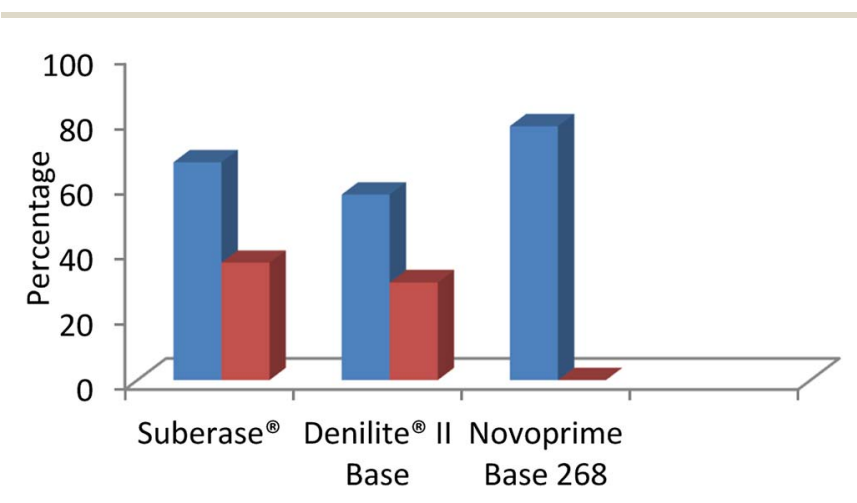

Fig. 4 Comparison of commercial laccase preparations in the chemo-selective synthesis of 2-benzimidazole (blue) and the byproduct 2,3-benzimidazole (red). 
Table 2 Novoprime-catalysed formation of benzimidazoles through reaction of 0 -phenylenediamines ( 1 equiv.) and benzaldehyde derivatives (1 equiv.) in acetonitrile and acetate buffer $(\mathrm{pH} 4.0)$ at room temperature ${ }^{a}$

\begin{tabular}{|c|c|c|c|c|c|}
\hline Entry & Amine & Benzaldehyde & Time & Product & Yield \% \\
\hline 1 & $\mathrm{R}=\mathrm{H}$ & $\mathrm{R}_{1}=2-\mathrm{OMe}$ & $24 \mathrm{~h}$ & $3 a$ & 69 \\
\hline 2 & $\mathrm{R}=\mathrm{H}$ & $\mathrm{R}_{1}=3-\mathrm{OH}$ & $24 \mathrm{~h}$ & $4 b$ & 82 \\
\hline 3 & $\mathrm{R}=\mathrm{H}$ & $\mathrm{R}_{1}=2,5$-diOMe & $24 \mathrm{~h}$ & $3 \mathbf{c}$ & 76 \\
\hline 4 & $\mathrm{R}=\mathrm{H}$ & $\mathrm{R}_{1}=3,4$-diOMe & $24 \mathrm{~h}$ & 3d & 78 \\
\hline 5 & $\mathrm{R}=\mathrm{H}$ & $\mathrm{R}_{1}=\mathrm{H}$ & $2 \mathrm{~h}$ & $3 \mathbf{e}$ & 92 \\
\hline 6 & $\mathrm{R}=\mathrm{H}$ & $\mathrm{R}_{1}=2-\mathrm{Cl}$ & $2 \mathrm{~h}$ & $3 f$ & 94 \\
\hline 7 & $\mathrm{R}=\mathrm{H}$ & $\mathrm{R}_{1}=4-\mathrm{Cl}$ & $4 \mathrm{~h}$ & $3 g$ & 88 \\
\hline 8 & $\mathrm{R}=\mathrm{H}$ & $\mathrm{R}_{1}=2-\mathrm{NO}_{2}$ & $8 \mathrm{~h}$ & $3 \mathbf{h}$ & 56 \\
\hline 9 & $\mathrm{R}=\mathrm{H}$ & $\mathrm{R}_{1}=2-($ Pyridin- $4-\mathrm{yl})$ & $2 \mathrm{~h}$ & $3 \mathbf{i}$ & 60 \\
\hline 10 & $\mathrm{R}=\mathrm{H}$ & $\mathrm{R}_{1}=3-\mathrm{NO}_{2}$ & $4 \mathrm{~h}$ & $3 \mathbf{j}$ & 95 \\
\hline 11 & $\mathrm{R}=\mathrm{H}$ & $\mathrm{R}_{1}=4-($ Dimethylamino $)$ & $8 \mathrm{~h}$ & $3 \mathbf{k}$ & 64 \\
\hline 12 & $\mathrm{R}=\mathrm{H}$ & $\mathrm{R}_{1}=4-\mathrm{NO}_{2}$ & $2 \mathrm{~h}$ & 31 & 94 \\
\hline 13 & $\mathrm{R}=\mathrm{H}$ & $\mathrm{R}_{1}=3,4,5$-TriOMe & $24 \mathrm{~h}$ & $3 \mathrm{~m}$ & 89 \\
\hline 14 & $\mathrm{R}=\mathrm{H}$ & $\mathrm{R}_{1}=4-\mathrm{OMe}$ & $24 \mathrm{~h}$ & $3 n$ & 68 \\
\hline 15 & $\mathrm{R}=4-\mathrm{Br}$ & $\mathrm{R}_{1}=2-($ Pyridin $-4-\mathrm{yl})$ & $4 \mathrm{~h}$ & 30 & 94 \\
\hline 16 & $\mathrm{R}=4-\mathrm{Cl}$ & $\mathrm{R}_{1}=3,4$-diOMe & $2 \mathrm{~h}$ & $3 p$ & 93 \\
\hline 17 & $\mathrm{R}=4-\mathrm{Me}$ & $\mathrm{R}_{1}=\mathrm{H}$ & $8 \mathrm{~h}$ & $3 q$ & 79 \\
\hline 18 & $\mathrm{R}=\mathrm{H}$ & $\mathrm{R}_{1}=2-\mathrm{Br}, 5-\mathrm{OMe}$ & $3 \mathrm{~h}$ & $3 \mathbf{r}$ & 90 \\
\hline
\end{tabular}

${ }^{a}$ Reaction conditions: laccase (0.105 g Novoprime Base 268) $50: 50$ acetonitrile/acetate buffer at room temperature.

molecule of benzaldehyde to afford another intermediate iminium ion ii. Successively, this intermediate may tautomerize to the most stable form iii to give the resultant disubstituted benzimidazole product $\mathbf{4 .}^{\mathbf{1 2}}$

In summary, we demonstrated that using acetonitrile in the presence of a catalytic amount of laccase (Novoprime Base 268) promotes the exclusive formation of the 2-phenyl-substituted benzimidazole for a broad range of derivatives. Our improved method is simple, selective and can afford 2-aryl- $1 H$-benzimidazoles in good to excellent yields.

For the synthesis of 2-arylbenzothiazoles, we began our investigation by exploring the oxidative condensation reaction between 2-aminothiophenol (5) and benzaldehyde (6a) under aerobic conditions at room temperature. The laccase-catalysed cross-coupling reaction between 5 and 6a to afford 2-phenylbenzothiazole (7a) (Fig. 5) in 85\% yield was performed in an acetate buffer (0.1 M, pH 4.0) using acetonitrile (50\%) as a cosolvent (Scheme 3 and Table 3, entry 4).

The effect of organic solvents such as ethanol, acetonitrile, hexane, chloroform, DMSO and water when preparing

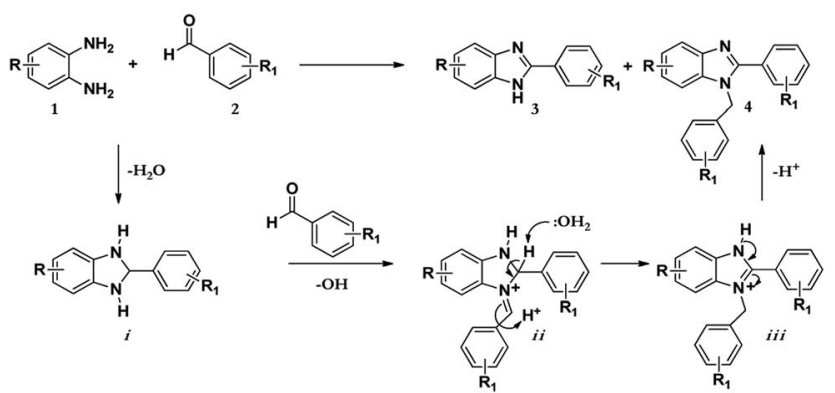

Scheme 2 Mechanism for the synthesis of the 1,2-disubstituted benzimidazoles. benzothiazoles have been investigated previously. ${ }^{37,38}$ Sayyahi et al. (2015) identified ethanol as the best solvent for preparing 2-arylbenzothiazoles under reflux conditions while Gao et al. (2014) described water as the optimal solvent using KI catalyst. $^{38,39}$ Therefore, in order to identify optimum reaction conditions we explored the effect of using various co-solvents. 2Aminothiophenol (5) and benzaldehyde (6a) were reacted in the presence of a laccase (Suberase ${ }^{\circledR}$, from Novozymes) in acetate buffer ( $\mathrm{pH}$ 4.0) using various solvents at room temperature (Table 3).

Acetonitrile gave the best yield under the described reaction conditions. While using methanol and ethanol as co-solvents, the reaction did not go to completion after $24 \mathrm{~h}$ of reaction and the product (7a) (yellow oil) was difficult to isolate from the starting material (2-aminothiophenol (5)) as they both have almost indistinguishable $R_{\mathrm{f}}$ values (40\% EtOAc/hexane). Conversely, Sayyahi et al. (2015) when using the ionic liquid [bmim $]\left[\mathrm{FeCl}_{4}\right]$ as a catalyst obtained excellent yields using ethanol but not acetonitrile. ${ }^{38}$

In order to purify compound 7a from the starting material, the product was dried overnight under high-vacuum to completely dry-out the oily product to an almost solid form, and cold acetonitrile was added to precipitate the product. An additional wash of cold acetonitrile $(3 \times 20.0 \mathrm{~mL})$ was used to remove residual starting material from the product.

To elucidate the role of the enzyme as a catalyst, control reactions were conducted using benzaldehydes $\mathbf{6 a - 1}$ under air in acetate buffer $(0.1 \mathrm{M}, \mathrm{pH} 4.0)$ /acetonitrile $(50: 50)$ in the absence of laccase (Scheme 4). However only two substrates resulted in the formation of a fused product in very low yield $(<10 \%)$ and no activity was observed for the remaining substrates (Table 4). Conversely, quantitative yields of the same substrates were obtained in the presence of an enzyme.

Numbers in brackets ( $7 \mathbf{b}$ and $7 \mathbf{e}$ ) - for these two compounds non-enzymatic limited background product formation that occurs in the absence of laccase was observed.

At this point, the most efficient method presenting the highest catalytic activity for preparing 2 -arylbenzothiazoles was using acetonitrile and acetate buffer ( $\mathrm{pH} 4.0)$, similar to the method described for 2-aryl-benzimidazole. Therefore to study

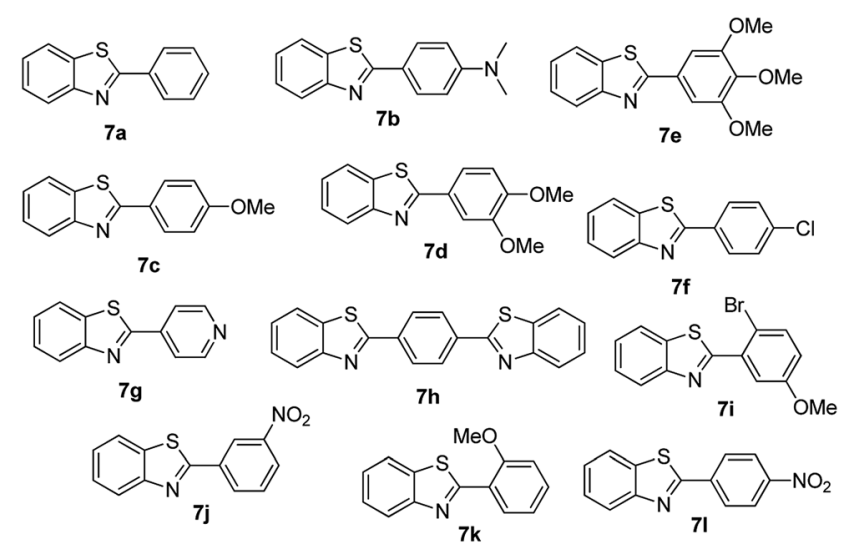

Fig. 5 Products $7 a-l$ of the laccase-catalysed domino reaction between 5 and $6 a-l$. 


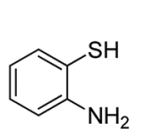

5

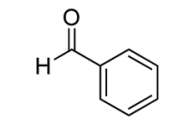

$6 a$

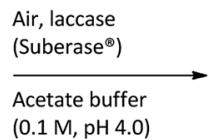

(0.1 M, pH 4.0)

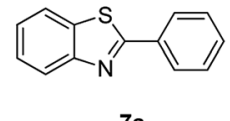

$7 a$
Scheme 3 Laccase-catalysed synthesis of 2-phenyl-benzothiazole.

Table 3 Suberase ${ }^{\circledR}$-catalysed synthesis of 2-phenyl-benzothiazole (7a) by reaction of 2-aminothiophenol (5) and benzaldehydes $6 a$ in acetate buffer $(0.1 \mathrm{M}, \mathrm{pH} 4.0)$ using various co-solvents ${ }^{a}$

\begin{tabular}{llllll}
\hline Entry & Aldehyde & Co-solvent & Time & Product & Yield \% \\
\hline 1 & $\mathbf{6 a}$ & Methanol & $24 \mathrm{~h}$ & $7 \mathbf{a}$ & 58 \\
2 & $\mathbf{6 a}$ & Ethanol & $24 \mathrm{~h}$ & $7 \mathbf{a}$ & 55 \\
3 & $\mathbf{6 a}$ & DMF & $24 \mathrm{~h}$ & $7 \mathbf{a}$ & 78 \\
4 & $\mathbf{6 a}$ & Acetonitrile & $24 \mathrm{~h}$ & $7 \mathbf{a}$ & 85 \\
5 & $\mathbf{6 a}$ & DCM & $24 \mathrm{~h}$ & $\mathbf{7 a}$ & 65
\end{tabular}

${ }^{a}$ Reaction conditions: Myceliophthora thermophilia laccase $(2.0 \mathrm{~mL}$ Suberase $\left.{ }^{\circledR}\right) 50: 50$ solvent/acetate buffer at $25{ }^{\circ} \mathrm{C}$.

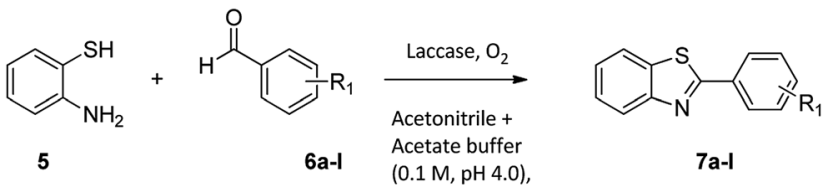

Scheme 4 Laccase-free synthesis of benzothiazoles.

the range and limitations of the optimised procedure 2-aminothiophenol was treated with a series of aryl-aldehydes in the presence of a catalytic amount of laccase to yield 2-arylbenzothiazole derivatives in good to excellent yield at room temperature (General method) and the results obtained are summarized in Table 4. Similar reactivity was observed for benzaldehydes with a variety of substituent groups; both electron withdrawing and electron donating at various positions showed comparable yields and reaction rates.

Table 4 Novoprime-catalysed synthesis of benzothiazoles through reaction of 1 equiv. of 2 -aminothiophenol (5) with 1 equiv. of benzaldehydes $6 \mathrm{a}-1$ in acetate buffer (0.1 M, pH 4.0)/acetonitrile (50:50) for $24 \mathrm{~h}$ via Scheme $3^{a}$

\begin{tabular}{|c|c|c|c|c|}
\hline Entry & Benzaldehyde & Time & Product & Yield \% \\
\hline 1 & $\mathrm{R}_{1}=\mathrm{H}$ & $24 \mathrm{~h}$ & $7 a$ & 85 \\
\hline 2 & $\begin{array}{l}\mathrm{R}_{1}=4^{-} \\
\text {(Dimethylamino) }\end{array}$ & $24 \mathrm{~h}$ & $7 \mathbf{b}$ & $56(8)$ \\
\hline 3 & $\mathrm{R}_{1}=4-\mathrm{OMe}$ & $24 \mathrm{~h}$ & $7 \mathrm{c}$ & 68 \\
\hline 4 & $\mathrm{R}_{1}=3$,4-diOMe & $24 \mathrm{~h}$ & $7 d$ & 73 \\
\hline 5 & $\mathrm{R}_{1}=3,4,5-\mathrm{TriOMe}$ & $24 \mathrm{~h}$ & $7 e$ & $65(7)$ \\
\hline 6 & $\mathrm{R}_{1}=4-\mathrm{Cl}$ & $24 \mathrm{~h}$ & $7 f$ & 56 \\
\hline 7 & $\mathrm{R}_{1}=2-($ Pyridin-4-yl $)$ & $24 \mathrm{~h}$ & $7 \mathrm{~g}$ & 88 \\
\hline 8 & $\mathrm{R}_{1}=4$-Acetyl & $24 \mathrm{~h}$ & $7 \mathbf{h}$ & 87 \\
\hline 9 & $\mathrm{R}_{1}=2-\mathrm{Br}-5-\mathrm{Ome}$ & $24 \mathrm{~h}$ & $7 \mathbf{i}$ & 76 \\
\hline 10 & $\mathrm{R}_{1}=3-\mathrm{NO}_{2}$ & $24 \mathrm{~h}$ & $7 \mathbf{j}$ & 48 \\
\hline 11 & $\mathrm{R}_{1}=2-\mathrm{OMe}$ & $24 \mathrm{~h}$ & $7 \mathbf{k}$ & 74 \\
\hline 12 & $\mathrm{R}_{1}=4-\mathrm{NO}_{2}$ & $24 \mathrm{~h}$ & 71 & 84 \\
\hline
\end{tabular}

${ }^{a}$ Reaction conditions: laccase (0.105 g Novoprime Base 268) $50: 50$ acetonitrile/acetate buffer at room temperature.
In conclusion, we have successfully developed a simple and efficient method for the synthesis of 2-arylbenzothiazole derivatives using an inexpensive commercial laccase as a catalyst at room temperature. The optimal conditions for conducting these reactions are using acetate buffer $(0.1 \mathrm{M}, \mathrm{pH} 4.5)$ and acetonitrile as a co-solvent.

\section{Experimental}

\section{General method used to synthesize 2-aryl-benzothiazole derivatives}

A mixture of 2-aminoaromatic $(10.0 \mathrm{mmol})$ and benzaldehyde derivative $(10.0 \mathrm{mmol})$ in acetonitrile $(10.0 \mathrm{~mL})$ and acetate buffer (10.0 mL, pH 4.0) was stirred at room for 5 minutes. Laccase was then added into the mixture and the contents were stirred for 2$24 \mathrm{~h}$. When the reaction completed the product precipitated from the solution and was extracted with ethyl acetate $(30.0 \mathrm{~mL})$ and water $(3 \times 20.0 \mathrm{~mL})$ and concentrated on a rotary evaporator. The product was washed several times with cold acetonitrile $(3 \times 20.0$ $\mathrm{mL}$ ) to remove any excess starting material.

\section{General}

All reagents and solvents were purchased from Sigma-Aldrich (South Africa) or Merck KGaA (South Africa). All solvents used for chromatographic separation were distilled before use to remove any impurities. All the chemical reagents were used as received without any further purification. Reactions were monitored by TLC carried out on Merck aluminium foil backed plates coated with silica gel (60 F254) and visualization was done under UV light. Purification of some of the compounds was done using Macherey-Nagel silica gel 60 (particle size $0.063 \mathrm{~mm}$ to $0.20 \mathrm{~mm}$ ) purchased from Merck. All the melting point recordings of the compounds were performed on a Stuart SMP10 instrument. Bruker 300 and $500 \mathrm{MHz}$ spectrometers were used to record both the ${ }^{1} \mathrm{H}$ and ${ }^{13} \mathrm{C}$ Nuclear Magnetic Resonance data using a suitable solvent at room temperature. Data processing of the spectra was done using MestreNova Software under license from Mestrelab Research, CA, USA. The resonant frequency for all spectra obtained are reported in parts per million relative to an internal standard, TMS, which appears at zero parts per million. Coupling constants are reported in Hertz. A Bruker Tensor-27 Fourier Transform spectrometer was used to perform infrared spectroscopy.

LC-MS: a $10 \mu \mathrm{L}$ of the sample was injected into the Dionex Ultimate 3000 UHPLC system (Thermo Scientific, Dionex, Sunnyvale, California, USA) and run through a loop for one minute at $50 \%$ solvent A consisting of $0.1 \%$ formic acid in $\mathrm{H}_{2} \mathrm{O}(\mathrm{v} / \mathrm{v})$ and $50 \%$ solvent $\mathrm{B}$ consisting of $0.1 \%$ formic acid in acetonitrile $(\mathrm{v} / \mathrm{v})$ at a flowrate of $0.3 \mathrm{~mL} \mathrm{~min}^{-1}$. High resolution mass spectra were recorded on a inked Bruker Compact Q-TOF mass spectrometer (Bruker Daltonics, Bremen, Germany) using an ESI positive source.

Myceliophthora thermophilia laccases (Suberase ${ }^{8} 400 \mathrm{U} \mathrm{g}^{-1}$ and Denilite ${ }^{\circledR}$ II Base; $800 \mathrm{U} \mathrm{g}^{-1}$ ) and Novoprime Base 268 (origin and units not defined) were obtained from Novozymes. 
The following methods were used for the synthesis of benzimidazoles

Method A. A mixture of $o$-phenylenediamine $(10.0 \mathrm{mmol}, 1$ equiv.) and benzaldehyde derivative (20.0 mmol, 2 equiv.) in acetonitrile $(10.0 \mathrm{~mL})$ and acetate buffer $(10.0 \mathrm{~mL}, \mathrm{pH} 4.5)$ was stirred at room temperature for 5 minutes. Suberase ${ }^{\circledR}(2.0 \mathrm{~mL})$ was added to the mixture and the contents were stirred until reaction completes (monitored by TLC). The product precipitates from the solution as the reaction proceeds and after completion the product was extracted with ethyl acetate $(30.0 \mathrm{~mL})$ and water $(3 \times 20.0 \mathrm{~mL})$ and concentrated on a rotary evaporator. The product was washed several times with cold acetonitrile $(3 \times 20.0$ $\mathrm{mL}$ ) to remove any excess starting material.

Method B. The same as Method A, except that methanol (2.0 $\mathrm{mL}$ ) was used instead of acetonitrile.

Method C. The same as Method A, except that ethanol (4.0 $\mathrm{mL}$ ) was used instead of acetonitrile.

Method D. The same as Method A, except that DMF (10.0 $\mathrm{mL}$ ) was used instead of acetonitrile.

Method E. A repetition of Method A using Denilite ${ }^{\circledR}$ II Base $(0.085 \mathrm{~g})$ and Novoprime Base $268(0.105 \mathrm{~g})$ laccases instead of Suberase ${ }^{\circledR}(2.0 \mathrm{~mL})$.

\section{Synthesis of 2-(2-methoxyphenyl)-1H-benzimidazole (3a)}

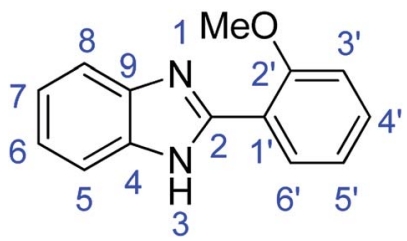

Method A. Stirring time $=24 \mathrm{~h}$. Purification by washing with cold acetonitrile $(3 \times 20.0 \mathrm{~mL})$ to remove any excess starting material (confirmed by TLC) to afford a brown solid (1.38 g, $62 \%) . R_{\mathrm{f}}(40 \%$ EtOAc/hexane) 0.38 .

Method B. Stirring time $=24 \mathrm{~h}$. Purification by washing with cold acetonitrile $(3 \times 20.0 \mathrm{~mL})$ to remove any excess starting material (confirmed by TLC) to afford a brown solid (1.32 g, $59 \%) . R_{\mathrm{f}}(40 \%$ EtOAc/hexane) 0.38 .

Method D. Stirring time $=24 \mathrm{~h}$. Purification by washing with cold acetonitrile $(3 \times 20.0 \mathrm{~mL})$ to remove any excess starting material (confirmed by TLC) to afford a brown solid (1.61 g, $72 \%) . R_{\mathrm{f}}$ (40\% EtOAc/hexane) 0.38 .

Method E. Novoprime Base $268(0.105 \mathrm{~g})$, stirring time $=$ $24 \mathrm{~h}$. Purification by washing with cold acetonitrile $(3 \times 20.0$ $\mathrm{mL}$ ) to remove any excess starting material (confirmed by TLC) to afford a brown solid $(1.55 \mathrm{~g}, 69 \%) . R_{\mathrm{f}}$ (40\% EtOAc/hexane) 0.38. $\mathrm{Mp}=178-180{ }^{\circ} \mathrm{C}$ (lit. $\left.40179-180{ }^{\circ} \mathrm{C}\right) .\left([\mathrm{M}+\mathrm{H}]^{+}\right.$found: 225.1023 $\mathrm{C}_{14} \mathrm{H}_{12} \mathrm{~N}_{2} \mathrm{O}$ requires $\left.[\mathrm{M}+\mathrm{H}]^{+}, 225.1024\right) .{ }^{1} \mathrm{H}$ NMR $(500$ MHz, DMSO- $\left.d_{6}\right): \delta 12.11(1 \mathrm{H}, \mathrm{s}, \mathrm{NH}), 8.33(1 \mathrm{H}, \mathrm{d}, J=7.6 \mathrm{~Hz}$, $\mathrm{H6}^{\prime}$ ), 7.63 (2H, dd, $\left.J=16.6,7.5 \mathrm{~Hz}, \mathrm{H} 5, \mathrm{H} 8\right), 7.48(1 \mathrm{H}, \mathrm{t}, J=$ $\left.7.8 \mathrm{~Hz}, \mathrm{H}^{\prime}\right), 7.24(1 \mathrm{H}, \mathrm{d}, J=8.3 \mathrm{~Hz}, \mathrm{H} 7), 7.19(2 \mathrm{H}, \mathrm{t}, J=6.5 \mathrm{~Hz}$, $\mathrm{H}^{\prime}, \mathrm{H}^{\prime}$ ), $7.12(1 \mathrm{H}, \mathrm{t}, J=7.5 \mathrm{~Hz}, \mathrm{H} 6), 4.03$ (3H, s, OMe). ${ }^{13} \mathrm{C}$ NMR (126 MHz, DMSO- $d_{6}$ ): $\delta 157.25$ (C-2), 149.43 (C-2'), 143.20

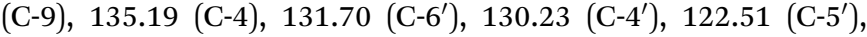
121.96 (C-1'), 121.34 (C-3'), 118.89 (C-7), 118.63 (C-6), 112.58 (C8), 112.38 (C-5), 56.25 (OMe). IR ( $\left.v_{\max } / \mathrm{cm}^{-1}\right): 3043$ (ArC-H); 1619 $(\mathrm{C}=\mathrm{N}) ; 1584,1537(\operatorname{ArC}=\mathrm{C}) ; 1306(\mathrm{C}-\mathrm{O})$.

\section{Synthesis of 2-(2,5-dimethoxyphenyl)-1H-benzimidazole (3c)}

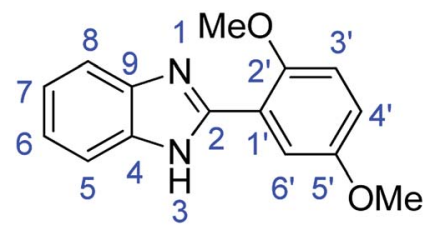

Method B. Stirring time $=24 \mathrm{~h}$. Purification by washing with cold acetonitrile $(3 \times 20.0 \mathrm{~mL})$ to remove any excess starting material (confirmed by TLC) to afford a brown solid $(0.253 \mathrm{~g}$, $10 \%) \cdot R_{\mathrm{f}}(40 \%$ EtOAc/hexane) 0.38 .

Method C. Stirring time $=24 \mathrm{~h}$. Purification by washing with cold acetonitrile $(3 \times 20.0 \mathrm{~mL})$ to remove any excess starting material (confirmed by TLC) to afford a brown solid (0.677 g, $25 \%) \cdot R_{\mathrm{f}}(40 \%$ EtOAc/hexane) 0.38 .

Method E. Novoprime Base 268 (0.105 g), stirring time = $24 \mathrm{~h}$. Purification by washing with cold acetonitrile $(3 \times 20.0$ $\mathrm{mL}$ ) to remove any excess starting material (confirmed by TLC) to afford a white solid (1.93 g, 76\%). $R_{\mathrm{f}}$ (40\% EtOAc/hexane) 0.38. $\mathrm{Mp}=224-226{ }^{\circ} \mathrm{C}$. $\left([\mathrm{M}+\mathrm{H}]^{+}\right.$found: $255.1126 \mathrm{C}_{15} \mathrm{H}_{14} \mathrm{~N}_{2} \mathrm{O}_{2}$ requires $\left.[\mathrm{M}+\mathrm{H}]^{+}, 255.1129\right)$. ${ }^{1} \mathrm{H}$ NMR (500 MHz, DMSO- $d_{6}$ ): $\delta 12.09(1 \mathrm{H}, \mathrm{s}, \mathrm{NH}), 7.87\left(1 \mathrm{H}, \mathrm{d}, J=2.8 \mathrm{~Hz}, \mathrm{H} 3^{\prime}\right), 7.63(2 \mathrm{H}, \mathrm{dd}, J=$ 19.0, 7.2 Hz, H5, H8), 7.19 (3H, m, H6, H7, H6'), 7.05 (1H, dd, J= 9.0, $2.9 \mathrm{~Hz}, \mathrm{H} 4^{\prime}$ ), 3.98 (3H, s, OMe), 3.81 (3H, s, OMe). ${ }^{13} \mathrm{C} \mathrm{NMR}$ (126 MHz, DMSO- $\left.d_{6}\right): \delta 153.69(\mathrm{C}-2), 151.56\left(\mathrm{C}^{\prime} 5^{\prime}\right), 149.21\left(\mathrm{C}-2^{\prime}\right)$, 143.10 (C-9), 135.23 (C-4), 122.61 (C-7), 122.04 (C-6), 118.92 (C$1^{\prime}$ ), 117.60 (C-4'), 114.10 (C-5, C-8), $113.96\left(\mathrm{C}-2^{\prime}\right), 112.48\left(\mathrm{C}-6^{\prime}\right)$, $56.65\left(\mathrm{OMe}^{\prime}\right), 56.03\left(\mathrm{OMe}^{\prime}\right)$. IR $\left(v_{\max } / \mathrm{cm}^{-1}\right): 2937(\mathrm{ArC}-\mathrm{H})$; $1619(\mathrm{C}=\mathrm{N}) ; 1523,1492(\mathrm{ArC}=\mathrm{C}) ; 1301(\mathrm{C}-\mathrm{O})$.

Synthesis of 2-(3,4-dimethoxyphenyl)-1H-benzimidazole (3d)

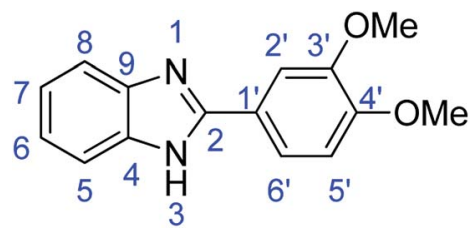

Method A. Stirring time $=24 \mathrm{~h}$. Purification by washing with cold acetonitrile $(3 \times 20.0 \mathrm{~mL})$ to remove any excess starting material (confirmed by TLC) to afford a brown solid (1.48 g, $59 \%) . R_{\mathrm{f}}\left(60 \%\right.$ EtOAc/hexane) 0.43. $\mathrm{Mp}=230-232{ }^{\circ} \mathrm{C}$ (lit. 41 231-232 $\left.{ }^{\circ} \mathrm{C}\right) \cdot\left([\mathrm{M}+\mathrm{H}]^{+}\right.$found: $255.1127 \mathrm{C}_{15} \mathrm{H}_{14} \mathrm{~N}_{2} \mathrm{O}_{2}$ requires $[\mathrm{M}$ $\left.+\mathrm{H}]^{+}, 255.1129\right) .{ }^{1} \mathrm{H}$ NMR (300 MHz, chloroform-d): $\delta$ 8.28-7.99 (1H, m, H8), 7.74-7.43 (3H, m, H5, H2' $\left.\mathrm{H}^{\prime}\right), 7.22(1 \mathrm{H}, \mathrm{d}, J=$ $8.2 \mathrm{~Hz}, \mathrm{H} 7), 7.14(1 \mathrm{H}, \mathrm{dd}, J=8.3,3.5 \mathrm{~Hz}, \mathrm{H} 6), 7.08-6.89(1 \mathrm{H}, \mathrm{m}$, $\left.\mathrm{H}^{\prime}\right)$, 5.75 (1H, s, NH), 4.85-3.50 (6H, m, $\left.2 \times \mathrm{OMe}\right) .{ }^{13} \mathrm{C} \mathrm{NMR}$ (126 MHz, DMSO- $\left.d_{6}\right): \delta 153.70(\mathrm{C}-2), 150.56\left(\mathrm{C}-3^{\prime}\right), 149.39\left(\mathrm{C}-4^{\prime}\right)$, 
143.09 (C-9), 136.51 (C-4), 129.86 (C-1'), 122.97 (C-7), 122.85 (C6), 122.51 (C-6'), 118.95 (C-8), 118.53 (C-5), 112.32 (C-2'), 111.38 $\left(\mathrm{C}-5^{\prime}\right), 56.10(2 \times \mathrm{OMe}): \operatorname{IR}\left(v_{\max } / \mathrm{cm}^{-1}\right): 2940(\mathrm{ArC}-\mathrm{H}) ; 1606(\mathrm{C}=$ $\mathrm{N})$; 1588, $1501(\operatorname{ArC}=\mathrm{C}) ; 1320(\mathrm{C}-\mathrm{O})$.

Method B. Stirring time $=24 \mathrm{~h}$. Purification by washing with cold acetonitrile $(3 \times 20.0 \mathrm{~mL})$ to remove any excess starting material (confirmed by TLC) to afford a brown solid $(0.864 \mathrm{~g}$, $34 \%) . R_{\mathrm{f}}(60 \%$ EtOAc/hexane) 0.42 .

Method D. Stirring time $=24 \mathrm{~h}$. Purification by washing with cold acetonitrile $(3 \times 20.0 \mathrm{~mL})$ to remove any excess starting material (confirmed by TLC) to afford a brown solid (1.71 g, $67 \%) . R_{\mathrm{f}}(60 \%$ EtOAc/hexane) 0.42 .

Method E. Denilite ${ }^{\circledR}$ II Base $(0.085 \mathrm{~g})$, stirring time $=24 \mathrm{~h}$. Purification by washing with cold acetonitrile $(3 \times 20.0 \mathrm{~mL})$ to remove any excess starting material (confirmed by TLC) to afford a yellow oil (1.45 g, 57\%). $R_{\mathrm{f}}(60 \%$ EtOAc/hexane) 0.42 .

Method E. Novoprime Base $268(0.105 \mathrm{~g})$, stirring time $=8 \mathrm{~h}$. Purification by washing with cold acetonitrile $(3 \times 20.0 \mathrm{~mL})$ to remove any excess starting material (confirmed by TLC) to afford a brown solid (1.97 g, 78\%). $R_{\mathrm{f}}$ (60\% EtOAc/hexane) 0.42 .

\section{Synthesis of 2-phenyl-1H-benzimidazole (3e)}

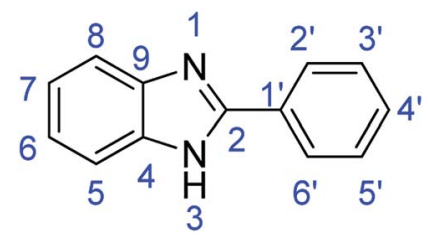

Method E. Novoprime Base 268 (0.105 g), stirring time $=2 \mathrm{~h}$. Purification by washing with cold acetonitrile $(3 \times 20.0 \mathrm{~mL})$ to remove any excess starting material (confirmed by TLC) to afford a brown solid $(1.77 \mathrm{~g}, 92 \%) . R_{\mathrm{f}}(40 \%$ EtOAc/hexane) $0.42 . \mathrm{Mp}=$ 290-293 ${ }^{\circ} \mathrm{C}$ (lit. $40292-293{ }^{\circ} \mathrm{C}$ ). $[\mathrm{M}+\mathrm{H}]^{+}$found: 195.0916 $\mathrm{C}_{13} \mathrm{H}_{10} \mathrm{~N}_{2}$ requires $\left.[\mathrm{M}+\mathrm{H}]^{+}, 195.0918\right) .{ }^{1} \mathrm{H}$ NMR $(500 \mathrm{MHz}$, DMSO- $\left.d_{6}\right): \delta 12.89(1 \mathrm{H}, \mathrm{s}, \mathrm{NH}), 8.19\left(2 \mathrm{H}, \mathrm{d}, J=7.5 \mathrm{~Hz}, \mathrm{H}^{\prime}, \mathrm{H}^{\prime}\right)$, $7.68(1 \mathrm{H}, \mathrm{d}, J=7.6 \mathrm{~Hz}, \mathrm{H} 8), 7.53$ (4H, m, H5, H7, H3' $\left.{ }^{\prime} \mathrm{H}^{\prime}\right), 7.21$ $\left(2 \mathrm{H}, \mathrm{dt}, J=15.6,6.6 \mathrm{~Hz}, \mathrm{H} 6, \mathrm{H} 44^{\prime}\right) .{ }^{13} \mathrm{C} \mathrm{NMR}\left(126 \mathrm{MHz}, \mathrm{DMSO}-d_{6}\right)$ : $\delta 151.69$ (C-2), 144.29 (C-9, C-4), 135.47 (C-1'), $130.65\left(\mathrm{C}-4^{\prime}\right)$, $130.29\left(\mathrm{C}-3^{\prime},{\mathrm{C}-5^{\prime}}^{\prime}\right), 129.40\left(\mathrm{C}-2^{\prime}\right), 126.90\left(\mathrm{C}^{-6}{ }^{\prime}\right), 122.99$ (C-7), 122.12 (C-6), 119.34 (C-8), 111.78 (C-5). IR $\left(v_{\max } / \mathrm{cm}^{-1}\right): 3047$ (ArC-H); $2921(\mathrm{~N}-\mathrm{H}) ; 1622(\mathrm{C}=\mathrm{N}) ; 1559,1542(\operatorname{ArC}=\mathrm{C})$.

\section{Synthesis of 2-(2-chlorophenyl)-1H-benzimidazole (3f)}

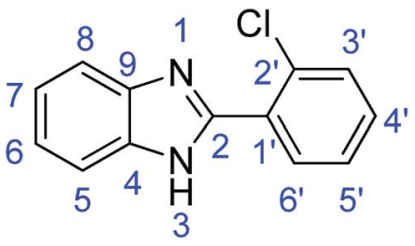

Method E. Novoprime Base 268 (0.105 g), stirring time $=2 \mathrm{~h}$. Purification by washing with cold acetonitrile $(3 \times 20.0 \mathrm{~mL})$ to remove any excess starting material (confirmed by TLC) to afford a brown solid (2.14 g, 94\%). $R_{\mathrm{f}}(40 \%$ EtOAc/hexane) 0.58 . $\mathrm{Mp}=231-234{ }^{\circ} \mathrm{C}$ (lit. $\left.40234-235^{\circ} \mathrm{C}\right) .\left([\mathrm{M}+\mathrm{H}]^{+}\right.$found: 229.0527 $\mathrm{C}_{13} \mathrm{H}_{9} \mathrm{ClN}_{2}$ requires $\left.[\mathrm{M}+\mathrm{H}]^{+}, 229.0528\right) .{ }^{1} \mathrm{H}$ NMR $(500 \mathrm{MHz}$, DMSO- $\left.d_{6}\right): \delta 12.71(1 \mathrm{H}, \mathrm{s}, \mathrm{NH}), 7.92\left(1 \mathrm{H}, \mathrm{d}, J=7.1 \mathrm{~Hz}, \mathrm{H}^{\prime}\right)$, 7.78-7.46 (5H, m, H5, H8, H3' $\left.{ }^{\prime} \mathrm{H}^{\prime}, \mathrm{H}^{\prime}\right), 7.25(2 \mathrm{H}, \mathrm{d}, J=6.8 \mathrm{~Hz}$, H6, H7). ${ }^{13} \mathrm{C}$ NMR (126 MHz, DMSO- $d_{6}$ ): $\delta 149.56$ (C-2), 143.69 (C-1'), 135.13 (C-9), 132.54 (C-4), 132.11 (C-2'), 131.65 (C-4'), $130.81\left(\mathrm{C}-3^{\prime}\right), 130.46\left(\mathrm{C}-6^{\prime}\right), 127.88\left(\mathrm{C}-5^{\prime}\right), 123.18$ (C-7), 122.17 (C6), 119.56 (C-8), 112.17 (C-5). IR $\left(v_{\max } / \mathrm{cm}^{-1}\right)$ : $3045(\mathrm{ArC}-\mathrm{H}) ; 1622$ $(\mathrm{C}=\mathrm{N}) ; 1569,1539(\mathrm{ArC}=\mathrm{C}) ; 700(\mathrm{C}-\mathrm{Cl})$.

\section{Synthesis of 2-(4-chlorophenyl)-1H-benzimidazole (3g)}

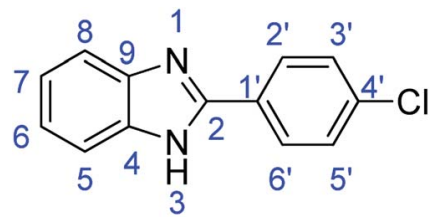

Method E. Novoprime Base 268 (0.105 g), stirring time $=4 \mathrm{~h}$. Purification by washing with cold acetonitrile $(3 \times 20.0 \mathrm{~mL})$ to remove any excess starting material (confirmed by TLC) to afford a brown solid (2.02 g, 88\%). $R_{\mathrm{f}}$ (40\% EtOAc/hexane) 0.42 . $\mathrm{Mp}=286-289^{\circ} \mathrm{C}$ (lit. $\left.42291-294^{\circ} \mathrm{C}\right) .\left([\mathrm{M}+\mathrm{H}]^{+}\right.$found: 229.0527 $\mathrm{C}_{13} \mathrm{H}_{9} \mathrm{ClN}_{2}$ requires $\left.[\mathrm{M}+\mathrm{H}]^{+}, 229.0528\right) .{ }^{1} \mathrm{H}$ NMR $(500 \mathrm{MHz}$, DMSO- $\left.d_{6}\right): \delta 12.98(1 \mathrm{H}, \mathrm{s}, \mathrm{NH}), 8.20\left(2 \mathrm{H}, \mathrm{d}, J=8.4 \mathrm{~Hz}, \mathrm{H}^{\prime}, \mathrm{H}^{\prime}\right)$, 7.64 (4H, m, H5, H8, H3' $\mathrm{H}^{\prime}$ ), 7.22 (2H, d, $\left.J=6.7 \mathrm{~Hz}, \mathrm{H} 6, \mathrm{H} 7\right)$. ${ }^{13} \mathrm{C}$ NMR (126 MHz, DMSO- $d_{6}$ ): $\delta 149.56$ (C-2), 143.69 (C-9), 135.13 (C-4), $132.54\left(\mathrm{C}-4^{\prime}\right), 132.11\left(\mathrm{C}^{\prime} \mathbf{1}^{\prime}\right), 131.65\left(\mathrm{C}-3^{\prime}\right), 130.81$ (C-5'), 130.46 (C-2'), 127.88 (C-6'), 123.18 (C-7), 122.17 (C-6), 119.56 (C-8), 112.17 (C-5). IR $\left(v_{\max } / \mathrm{cm}^{-1}\right): 3052(\mathrm{ArC}-\mathrm{H}) ; 1622$ $(\mathrm{C}=\mathrm{N}) ; 1588,1540(\mathrm{ArC}=\mathrm{C}) ; 727(\mathrm{C}-\mathrm{Cl})$.

Synthesis of 2-(2-nitrophenyl)-1H-benzimidazole (3h)

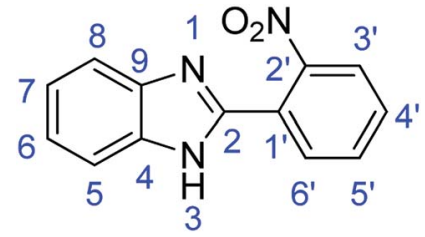

Method E. Novoprime Base 268 (0.105 g), stirring time $=8 \mathrm{~h}$. Purification by washing with cold acetonitrile $(3 \times 20.0 \mathrm{~mL})$ to remove any excess starting material (confirmed by TLC) to afford an orange-brown solid (1.33 g, 56\%). $R_{\mathrm{f}}(40 \%$ EtOAc/ hexane) 0.22. $\mathrm{Mp}=191-193{ }^{\circ} \mathrm{C}$ (lit. 43 190-193 ${ }^{\circ} \mathrm{C}$ ). ${ }^{1} \mathrm{H}$ NMR $\left(500 \mathrm{MHz}, \mathrm{DMSO}-d_{6}\right): \delta 12.21(1 \mathrm{H}, \mathrm{s}, \mathrm{NH}), 7.21(1 \mathrm{H}, \mathrm{d}, J=8.0 \mathrm{~Hz}$, $\left.\mathrm{H6}^{\prime}\right), 7.15\left(1 \mathrm{H}, \mathrm{d}, J=7.7 \mathrm{~Hz}, \mathrm{H} 3^{\prime}\right), 7.04\left(1 \mathrm{H}, \mathrm{t}, J=7.6 \mathrm{~Hz}, \mathrm{H}^{\prime}\right)$, $6.94\left(1 \mathrm{H}, \mathrm{t}, J=7.7 \mathrm{~Hz}, \mathrm{H} 4^{\prime}\right), 6.79(2 \mathrm{H}, \mathrm{s}, \mathrm{H} 5, \mathrm{H} 8), 6.43(2 \mathrm{H}, \mathrm{s}, \mathrm{H} 6$, H7). ${ }^{13} \mathrm{C}$ NMR (126 MHz, DMSO- $\left.d_{6}\right): \delta 149.44$ (C-2), 147.77 (C-2'), 133.11 (C-4, C-9), 131.39 (C-5', C-6'), 131.36 (C-1', C-4'), 124.76 (C-6, C-7, C-3'), 124.71 (C-5, C-8). IR $\left(v_{\max } / \mathrm{cm}^{-1}\right): 3064$ (ArC-H); $1625(\mathrm{C}=\mathrm{N}) ; 1573,1524(\operatorname{ArC}=\mathrm{C})$. 
Synthesis of 2-(pyridin-4-yl)-1H-benzimidazole (3i)

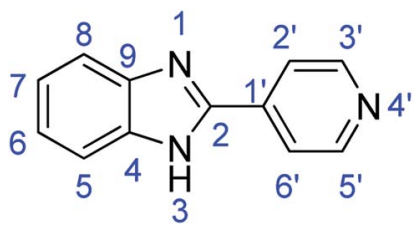

Method E. Novoprime Base 268 (0.105 g), stirring time $=2 \mathrm{~h}$. Purification by washing with cold acetonitrile $(3 \times 20.0 \mathrm{~mL})$ to remove any excess starting material (confirmed by TLC) to afford a brown solid (1.69 g, 60\%). $R_{\mathrm{f}}(40 \%$ EtOAc/hexane) 0.13 . $\mathrm{Mp}=211-214{ }^{\circ} \mathrm{C}$ (lit. $\left.42214{ }^{\circ} \mathrm{C}\right) .\left([\mathrm{M}+\mathrm{H}]^{+}\right.$found: 196.0872 $\mathrm{C}_{12} \mathrm{H}_{9} \mathrm{~N}_{3}$ requires $\left.[\mathrm{M}+\mathrm{H}]^{+}, 196.0870\right) .{ }^{1} \mathrm{H} \mathrm{NMR}(500 \mathrm{MHz}$, DMSO- $\left.d_{6}\right): \delta 13.23(1 \mathrm{H}, \mathrm{s}, \mathrm{NH}), 8.76\left(2 \mathrm{H}, \mathrm{d}, J=4.9 \mathrm{~Hz}, \mathrm{H}^{\prime}, \mathrm{H}^{\prime}\right)$, $8.10\left(2 \mathrm{H}, \mathrm{d}, J=4.8 \mathrm{~Hz}, \mathrm{H} 2^{\prime}, \mathrm{H}^{\prime}\right), 7.66(2 \mathrm{H}, \mathrm{d}, J=68.5 \mathrm{~Hz}, \mathrm{H} 5$, H8), 7.50-7.02 (2H, m, H6, H7). ${ }^{13} \mathrm{C}$ NMR (126 MHz, DMSO- $\left.d_{6}\right)$ : $\delta 150.97$ (C-2), 149.24 (C-3', C-5'), 144.13 (C-1'), 137.60 (C-9), 135.49 (C-4), 124.03 (C-6, C-7), 122.73 (C-2'), 120.79 (C-6'), 119.93 (C-8), 112.27 (C-5). IR $\left(v_{\text {max }} / \mathrm{cm}^{-1}\right): 3051$ (ArC-H); 2877 $(\mathrm{N}-\mathrm{H}) ; 1665(\mathrm{C}=\mathrm{N}) ; 1562,1537(\mathrm{ArC}=\mathrm{C})$.

\section{Synthesis of 2-(3-nitrophenyl)-1 $\mathrm{H}$-benzimidazole $(3 \mathbf{j})$}

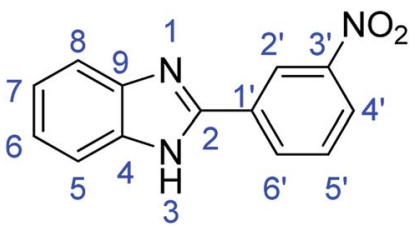

Method E. Novoprime Base 268 (0.105 g), stirring time $=4 \mathrm{~h}$. Purification by washing with cold acetonitrile $(3 \times 20.0 \mathrm{~mL})$ to remove any excess starting material (confirmed by TLC) to afford a brown solid $(2.26 \mathrm{~g}, 95 \%) . R_{\mathrm{f}}$ (40\% EtOAc/hexane) 0.43 . $\mathrm{Mp}=202-205{ }^{\circ} \mathrm{C}$ (lit. $\left.43201-203{ }^{\circ} \mathrm{C}\right) .\left([\mathrm{M}+\mathrm{H}]^{+}\right.$found: 196.0872 $\mathrm{C}_{13} \mathrm{H}_{9} \mathrm{ClN}_{2}$ requires $\left.[\mathrm{M}+\mathrm{H}]^{+}, 196.0870\right) .{ }^{1} \mathrm{H}$ NMR $(500 \mathrm{MHz}$, DMSO- $\left.d_{6}\right): \delta 13.27(1 \mathrm{H}, \mathrm{s}, \mathrm{NH}), 9.02\left(1 \mathrm{H}, \mathrm{s}, \mathrm{H} 2^{\prime}\right), 8.62(1 \mathrm{H}, \mathrm{d}, J=$ $\left.7.7 \mathrm{~Hz}, \mathrm{H6}^{\prime}\right), 8.33\left(1 \mathrm{H}, \mathrm{d}, J=8.2 \mathrm{~Hz}, \mathrm{H} 4^{\prime}\right), 7.86(1 \mathrm{H}, \mathrm{t}, J=8.0 \mathrm{~Hz}$, $\left.\mathrm{H}^{\prime}\right), 7.66(2 \mathrm{H}, \mathrm{d}, J=50.7 \mathrm{~Hz}, \mathrm{H} 5, \mathrm{H} 8), 7.26(2 \mathrm{H}, \mathrm{d}, J=6.7 \mathrm{~Hz}$, H6, H7). ${ }^{13} \mathrm{C}$ NMR (126 MHz, DMSO- $d_{6}$ ): $\delta 147.43$ (C-2), 146.75 (C-3'), 130.84 (C-4, C-9), 130.12 (C-6'), 129.04 (C-1', C-5'), 122.55 (C-4'), 119.20 (C-6, C-7, C-2'), 116.36 (C-5, C-8). IR ( $\left.v_{\max } / \mathrm{cm}^{-1}\right)$ : $3098(\operatorname{ArC}-\mathrm{H}) ; 1623(\mathrm{C}=\mathrm{N}) ; 1588,1517(\operatorname{ArC}=\mathrm{C})$.

\section{Synthesis of 4-(1H-benzimidazol-2-yl)- $N, N$-dimethylaniline (3k)}

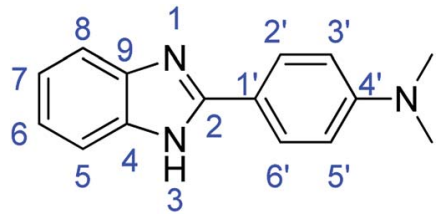

\section{Method E}

Novoprime Base 268 (0.105 g), stirring time $=8 \mathrm{~h}$. Purification by washing with cold acetonitrile $(3 \times 20.0 \mathrm{~mL})$ to remove any excess starting material (confirmed by TLC) to afford an orange solid (1.52 g, 64\%). $R_{\mathrm{f}}$ (40\% EtOAc/hexane) 0.17. Mp $=201-$ $202{ }^{\circ} \mathrm{C}$ (lit. $\left.44202-204{ }^{\circ} \mathrm{C}\right) \cdot\left([\mathrm{M}+\mathrm{H}]^{+}\right.$found: $238.1339 \mathrm{C}_{15} \mathrm{H}_{15} \mathrm{~N}_{3}$ requires $\left.[\mathrm{M}+\mathrm{H}]^{+}, 238.1340\right) .{ }^{1} \mathrm{H}$ NMR (500 MHz, DMSO- $\left.d_{6}\right)$ : $\delta 8.14(1 \mathrm{H}, \mathrm{s}, \mathrm{NH}), 7.99\left(2 \mathrm{H}, \mathrm{d}, J=8.7 \mathrm{~Hz}, \mathrm{H} 2^{\prime}, \mathrm{H}^{\prime}\right), 7.48(2 \mathrm{H}, \mathrm{d}, J$ $=9.2 \mathrm{~Hz}, \mathrm{H} 5, \mathrm{H} 8), 7.14(2 \mathrm{H}, \mathrm{d}, J=9.2 \mathrm{~Hz}, \mathrm{H} 6, \mathrm{H} 7), 6.83(2 \mathrm{H}, \mathrm{d}, J$ $\left.=8.7 \mathrm{~Hz}, \mathrm{H}^{\prime}, \mathrm{H}^{\prime}\right), 3.00\left(6 \mathrm{H}, \mathrm{s}, 2 \times \mathrm{N}-\mathrm{CH}_{3}\right) .{ }^{13} \mathrm{C} \mathrm{NMR}(126 \mathrm{MHz}$, DMSO- $\left.d_{6}\right): \delta 163.47$ (C-2), $152.72\left(\mathrm{C}-4^{\prime}\right), 151.74$ (C-9, C-4), 128.02 (C-2', C-6'), 121.80 (C-6, C-7), 117.83 (C-5, C-8, C-1'), 112.32 (C-3', $\left.\mathrm{C}-5^{\prime}\right)$. IR $\left(v_{\max } / \mathrm{cm}^{-1}\right): 2887(\mathrm{ArC}-\mathrm{H}) ; 2773(\mathrm{~N}-\mathrm{H}) ; 1637(\mathrm{C}=\mathrm{N})$; 1593, $1504(\operatorname{ArC}=\mathrm{C})$.

Synthesis of 2-(4-nitrophenyl)-1H-benzimidazole (3m)

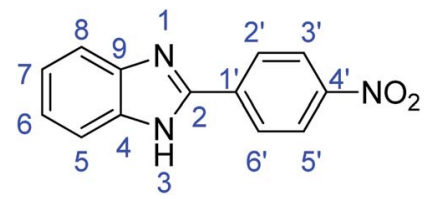

Method E. Novoprime Base 268 (0.105 g), stirring time $=2 \mathrm{~h}$. Purification by washing with cold acetonitrile $(3 \times 20.0 \mathrm{~mL})$ to remove any excess starting material (confirmed by TLC) to afford a brown solid (2.27 g, 95\%). $R_{\mathrm{f}}(40 \%$ EtOAc/hexane) 0.52 . $\mathrm{Mp}=320{ }^{\circ} \mathrm{C}$ (lit. $\left.40>300{ }^{\circ} \mathrm{C}\right)$. $\left([\mathrm{M}+\mathrm{H}]^{+}\right.$found: 240.0767 $\mathrm{C}_{13} \mathrm{H}_{9} \mathrm{~N}_{3} \mathrm{O}_{2}$ requires $\left.[\mathrm{M}+\mathrm{H}]^{+}, 240.0769\right) .{ }^{1} \mathrm{H}$ NMR $(500 \mathrm{MHz}$, DMSO- $\left.d_{6}\right): \delta 13.28(1 \mathrm{H}, \mathrm{s}, \mathrm{NH}), 8.42\left(4 \mathrm{H}, \mathrm{s}, \mathrm{H} 2^{\prime}, \mathrm{H} 3^{\prime}, \mathrm{H}^{\prime}, \mathrm{H}^{\prime}\right)$, $7.74(1 \mathrm{H}, \mathrm{d}, J=7.6 \mathrm{~Hz}, \mathrm{H} 8), 7.59(1 \mathrm{H}, \mathrm{d}, J=7.6 \mathrm{~Hz}, \mathrm{H} 5), 7.28$ $(2 \mathrm{H}, \mathrm{m}, \mathrm{H} 6, \mathrm{H} 7) .{ }^{13} \mathrm{C}$ NMR (126 MHz, DMSO- $\left.d_{6}\right): \delta 149.48(\mathrm{C}-2)$, 148.30 (C-4'), 144.32 (C-1'), 136.53 (C-9), 135.71 (C-4), 127.88 (C$\left.2^{\prime}, \mathrm{C}^{\prime} 6^{\prime}\right), 124.79\left(\mathrm{C}-3^{\prime}, \mathrm{C}-5^{\prime}\right), 124.07$ (C-7), 122.80 (C-6), 119.94 (C8), 112.28 (C-5). IR $\left(v_{\max } / \mathrm{cm}^{-1}\right): 2748(\mathrm{ArC}-\mathrm{H}) ; 1604(\mathrm{C}=\mathrm{N})$; 1513, $1433(\mathrm{ArC}=\mathrm{C})$.

Synthesis of 2-(3,4,5-trimethoxyphenyl)-1H-benzoimidazole (3n)

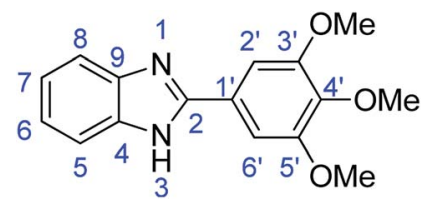

Method E. Novoprime Base 268 (0.105 g), stirring time = 24 h. Purification by washing with cold acetonitrile $(3 \times 20.0$ $\mathrm{mL}$ ) to remove any excess starting material (confirmed by TLC) to afford a white solid $(2.53 \mathrm{~g}, 89 \%) . R_{\mathrm{f}}$ (40\% EtOAc/hexane) 0.19. $\mathrm{Mp}=258-262{ }^{\circ} \mathrm{C}$ (lit. $\left.40258.7-259.7{ }^{\circ} \mathrm{C}\right) \cdot\left([\mathrm{M}+\mathrm{H}]^{+}\right.$ found: $285.1230 \mathrm{C}_{16} \mathrm{H}_{16} \mathrm{~N}_{2} \mathrm{O}_{3}$ requires $[\mathrm{M}+\mathrm{H}]^{+}$, 285.1224). ${ }^{1} \mathrm{H}$ NMR (500 MHz, DMSO- $\left.d_{6}\right): \delta 12.82(1 \mathrm{H}, \mathrm{s}, \mathrm{NH}), 7.66(1 \mathrm{H}, \mathrm{d}, J=$ $7.8 \mathrm{~Hz}, \mathrm{H} 8), 7.55-7.53$ (3H, m, H5, H6, H7), 7.20 (2H, p, $J=$ $\left.7.0 \mathrm{~Hz}, \mathrm{H} 2{ }^{\prime}, \mathrm{H}^{\prime}\right), 3.91$ (6H, s, $\left.2 \times \mathrm{OMe}\right), 3.74(3 \mathrm{H}, \mathrm{s}, \mathrm{OMe}) .{ }^{13} \mathrm{C}$ NMR (126 MHz, DMSO- $d_{6}$ ): $\delta 153.69$ (C-2), 151.68 (C-3', C-5'), 
144.22 (C-9), 139.38 (C-4), 135.44 (C-4'), 125.95 (C-1'), 122.89 (C7), 122.09 (C-6), 119.17 (C-5), 111.61 (C-8), 104.30 (C-2', C-6'), $60.62(\mathrm{OMe}), 56.53(2 \times \mathrm{OMe})$. IR $\left(v_{\max } / \mathrm{cm}^{-1}\right): 2952(\mathrm{ArC}-\mathrm{H})$; $1589(\mathrm{C}=\mathrm{N}) ; 1498,1482(\operatorname{ArC}=\mathrm{C}) ; 1311(\mathrm{C}-\mathrm{O})$.

\section{Synthesis of 2-(4-methoxyphenyl)-1H-benzimidazole (3o)}

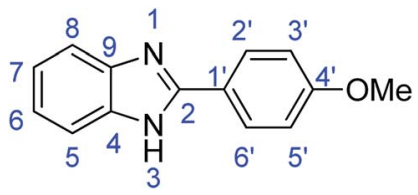

Method E. Novoprime Base 268 (0.105 g), stirring time = $24 \mathrm{~h}$. Purification by washing with cold acetonitrile $(3 \times 20.0$ $\mathrm{mL}$ ) to remove any excess starting material (confirmed by TLC) to afford a light brown solid $(1.53 \mathrm{~g}, 68 \%) . R_{\mathrm{f}}(40 \%$ EtOAc/hexane) 0.31. $\mathrm{Mp}=226-228{ }^{\circ} \mathrm{C}$ (lit. $\left.42225-226^{\circ} \mathrm{C}\right) .([\mathrm{M}$ $+\mathrm{H}]^{+}$found: $285.1230 \mathrm{C}_{16} \mathrm{H}_{16} \mathrm{~N}_{2} \mathrm{O}_{3}$ requires $[\mathrm{M}+\mathrm{H}]^{+}$, 285.1024) ${ }^{1} \mathrm{H}$ NMR (500 MHz, DMSO- $\left.d_{6}\right): \delta 12.73(1 \mathrm{H}, \mathrm{s}, \mathrm{NH})$, $8.12\left(2 \mathrm{H}, \mathrm{d}, J=8.6 \mathrm{~Hz}, \mathrm{H} 2^{\prime}, \mathrm{H}^{\prime}\right), 7.56(2 \mathrm{H}, \mathrm{d}, J=52.8 \mathrm{~Hz}, \mathrm{H} 5$, H8), 7.20-7.14 (2H, m, H6, H7), $7.11\left(2 \mathrm{H}, \mathrm{d}, J=8.6 \mathrm{~Hz}, \mathrm{H} 3^{\prime}\right.$, $\left.\mathrm{H}^{\prime}\right), 3.84$ (3H, s, OMe). ${ }^{13} \mathrm{C}$ NMR (126 MHz, DMSO- $\left.d_{6}\right)$ : $\delta 161.07$ (C-2), $151.82\left(\mathrm{C}-4^{\prime}\right), 144.37$ (C-9), 135.44 (C-4), 128.48 (C-2', C-6'), 123.19 (C-7), 122.49 (C-6), 121.98 (C-8), 121.91 (C-

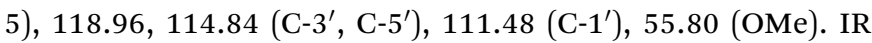
$\left(v_{\max } / \mathrm{cm}^{-1}\right): 3044(\operatorname{ArC}-\mathrm{H}) ; 1621(\mathrm{C}=\mathrm{N}) ; 1583,1500(\operatorname{ArC}=\mathrm{C})$; 1317 (C-O).

\section{Synthesis of 1-(2-methoxybenzyl)-2-(2-methoxyphenyl)-1H-} benzimidazole (4a)

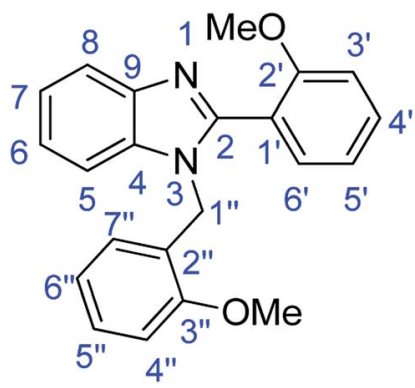

Method B. Stirring time $=24 \mathrm{~h}$. Purification by washing with cold acetonitrile $(3 \times 20.0 \mathrm{~mL})$ to remove any excess starting material (confirmed by TLC) to afford an orange solid ( $0.689 \mathrm{~g}$, $20 \%$ ). $R_{\mathrm{f}}$ (40\% EtOAc/hexane) 0.34. $\mathrm{Mp}=148-152{ }^{\circ} \mathrm{C}$ (lit. 44 $\left.151-153{ }^{\circ} \mathrm{C}\right) \cdot\left([\mathrm{M}+\mathrm{H}]^{+}\right.$found: $345.1596 \mathrm{C}_{22} \mathrm{H}_{20} \mathrm{~N}_{2} \mathrm{O}_{2}$ requires $[\mathrm{M}$ $\left.+\mathrm{H}]^{+}, 334.1599\right) .{ }^{1} \mathrm{H}$ NMR (500 MHz, DMSO- $\left.d_{6}\right): \delta 7.67(1 \mathrm{H}, \mathrm{d}, J$ $\left.=7.7 \mathrm{~Hz}, \mathrm{H6}^{\prime}\right), 7.52\left(1 \mathrm{H}, \mathrm{t}, J=7.8 \mathrm{~Hz}, \mathrm{H}^{\prime}\right), 7.42(1 \mathrm{H}, \mathrm{d}, J=$ $\left.7.4 \mathrm{~Hz}, \mathrm{H}^{\prime \prime}\right), 7.36\left(1 \mathrm{H}, \mathrm{d}, J=7.1 \mathrm{~Hz}, \mathrm{H}^{\prime}\right), 7.27-7.12(4 \mathrm{H}, \mathrm{m}, \mathrm{H} 6$, H7, H8, H4 $\left.4^{\prime}\right), 7.07\left(1 \mathrm{H}, \mathrm{t}, J=7.4 \mathrm{~Hz}, \mathrm{H}^{\prime \prime}\right), 6.92(1 \mathrm{H}, \mathrm{d}, J=8.2 \mathrm{~Hz}$, H5), $6.75\left(1 \mathrm{H}, \mathrm{t}, J=7.4 \mathrm{~Hz}, \mathrm{H}^{\prime \prime}\right), 6.58\left(1 \mathrm{H}, \mathrm{d}, J=7.4 \mathrm{~Hz}, \mathrm{H} 4^{\prime \prime}\right)$, 5.21 (2H, s, H1 ${ }^{\prime \prime}$ ), 3.67 (6H, s, OMe). ${ }^{13} \mathrm{C}$ NMR (126 MHz, DMSO- $\left.d_{6}\right): \delta 157.53\left(\mathrm{C}-3^{\prime \prime}\right), 156.83\left(\mathrm{C}-2^{\prime}\right), 152.31(\mathrm{C}-2), 143.35(\mathrm{C}-4)$, 135.68 (C-9), 132.39 (C-6'), 132.02 (C-4'), 129.17 (C-7"), 127.94 (C-5"), 124.64 (C-2"), 122.66 (C-7), 122.03 (C-6), 120.98 (C-5'), 120.57 (C-6"), 120.00 (C-1'), 119.54 (C-8), 111.96 (C-5), 111.33 (C$\left.3^{\prime}\right), 111.16$ (C-4"), 55.75 (OMe), 55.73 (OMe), 43.25 (C-1"). IR $\left(v_{\max } / \mathrm{cm}^{-1}\right): 3063(\operatorname{ArC}-\mathrm{H}) ; 1603(\mathrm{C}=\mathrm{N}) ; 1582,1518(\mathrm{ArC}=\mathrm{C})$; 1327 (C-O).

Method C. Stirring time $=24 \mathrm{~h}$. Purification by washing with cold acetonitrile $(3 \times 20.0 \mathrm{~mL})$ to remove any excess starting material (confirmed by TLC) to afford a brown solid (0.447 g, $13 \%) \cdot R_{\mathrm{f}}(40 \%$ EtOAc/hexane) 0.34 .

\section{Synthesis of 3-(1-(3-hydroxybenzyl)-1H-benzoimidazol-2-yl) phenol (4b)}

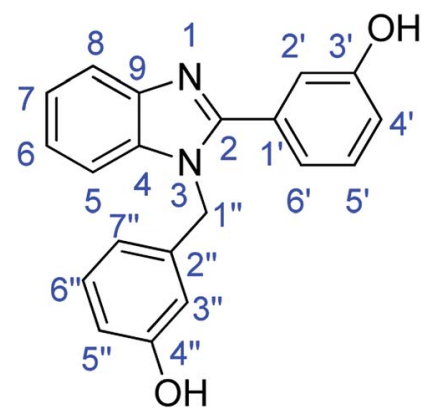

Method A. Stirring time $=24 \mathrm{~h}$. Purification by washing with cold acetonitrile $(3 \times 20.0 \mathrm{~mL})$ to remove any excess starting material (confirmed by TLC) to afford a brown solid $(2.58 \mathrm{~g}$, $82 \%) . R_{\mathrm{f}}\left(40 \%\right.$ EtOAc/hexane) 0.23. $\mathrm{Mp}=246-249{ }^{\circ} \mathrm{C}$ (lit. 45 243-245 $\left.{ }^{\circ} \mathrm{C}\right)$. ([M $\left.+\mathrm{H}\right]^{+}$found: $316 \mathrm{C}_{20} \mathrm{H}_{16} \mathrm{~N}_{2} \mathrm{O}_{2}$ requires $[\mathrm{M}+\mathrm{H}]^{+}$, 316.1286). ${ }^{1} \mathrm{H}$ NMR (500 MHz, DMSO- $\left.d_{6}\right): \delta 9.77(1 \mathrm{H}, \mathrm{d}, J=$ $30.8 \mathrm{~Hz}, \mathrm{H6}^{\prime}$ ), 9.43 (1H, s, H2'), 7.73 (1H, d, J = 7.7 Hz, H8), 7.39 $(1 \mathrm{H}, \mathrm{d}, J=7.5 \mathrm{~Hz}, \mathrm{H} 5), 7.33\left(1 \mathrm{H}, \mathrm{q}, J=7.7 \mathrm{~Hz}, \mathrm{H} 5^{\prime}\right), 7.23(3 \mathrm{H}, \mathrm{td}$, $\left.J=17.7,16.0,8.7 \mathrm{~Hz}, \mathrm{H} 6, \mathrm{H} 7, \mathrm{H}^{\prime \prime}\right), 7.17-7.06$ (2H, m, OH), 6.95 $\left(1 \mathrm{H}, \mathrm{d}, J=8.1 \mathrm{~Hz}, \mathrm{H}^{\prime \prime}\right), 6.65\left(1 \mathrm{H}, \mathrm{d}, J=8.0 \mathrm{~Hz}, \mathrm{H}^{\prime \prime}\right), 6.50(1 \mathrm{H}, \mathrm{d}$, $\left.J=7.5 \mathrm{~Hz}, \mathrm{H} 4^{\prime}\right), 6.40\left(1 \mathrm{H}, \mathrm{s}, \mathrm{H} 3^{\prime \prime}\right), 5.49\left(2 \mathrm{H}, \mathrm{s}, \mathrm{H} 1^{\prime \prime}\right) .{ }^{13} \mathrm{C} \mathrm{NMR}$ (126 MHz, DMSO- $d_{6}$ ): $\delta 156.09\left(\mathrm{C}-3^{\prime}\right), 155.91\left(\mathrm{C}-4^{\prime \prime}\right), 151.69(\mathrm{C}-2)$, 140.98 (C-4), 136.71 (C-9, C-2"), 134.25 (C-1'), $129.58\left(\mathrm{C}-5^{\prime}\right)$, 128.19 (C-6"), 120.96 (C-7), 120.52 (C-6), 117.87 (C-6'), 117.55 (C$\left.7^{\prime \prime}\right), 115.28$ (C-8), $114.99(\mathrm{C}-5), 114.38\left(\mathrm{C}-4^{\prime}\right), 112.82\left(\mathrm{C}-3^{\prime \prime}\right), 111.07$ $\left(\mathrm{C}-2^{\prime}\right), 109.45\left(\mathrm{C}-5^{\prime \prime}\right), 45.81\left(\mathrm{C}-1^{\prime \prime}\right)$.

Method C. Stirring time $=24 \mathrm{~h}$. Purification by washing with cold acetonitrile $(3 \times 20.0 \mathrm{~mL})$ to remove any excess starting material (confirmed by TLC) to afford a brown solid (3.06 g, $97 \%) . R_{\mathrm{f}}$ (40\% EtOAc/hexane) 0.23 .

Method D. Stirring time $=24 \mathrm{~h}$. Purification by washing with cold acetonitrile $(3 \times 20.0 \mathrm{~mL})$ to remove any excess starting material (confirmed by TLC) to afford a brown solid (3.10 g, $98 \%) \cdot R_{\mathrm{f}}$ (40\% EtOAc/hexane) 0.23 .

Method E. Novoprime Base 268 (0.105 g), stirring time $=4 \mathrm{~h}$. Purification by washing with cold acetonitrile $(3 \times 20.0 \mathrm{~mL})$ to remove any excess starting material (confirmed by TLC) to afford a brown solid $(2.72 \mathrm{~g}, 86 \%) . R_{\mathrm{f}}(40 \%$ EtOAc/hexane) 0.23 . 
Synthesis of 1-(2,5-dimethoxybenzyl)-2-(2,5-dimethoxyphenyl)$1 \mathrm{H}$-benzimidazole (4c)

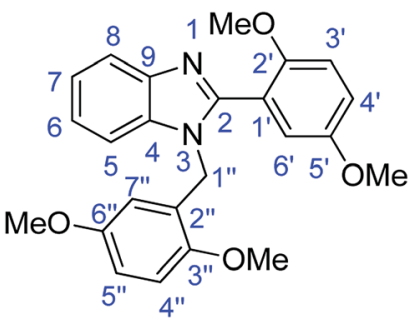

Method A. Stirring time $=24 \mathrm{~h}$. Purification by washing with cold acetonitrile $(3 \times 20.0 \mathrm{~mL})$ to remove any excess starting material (confirmed by TLC) to afford an oil $(2.25 \mathrm{~g}, 56 \%) . R_{\mathrm{f}}$ (40\% EtOAc/hexane) 0.36. ([M $+\mathrm{H}]^{+}$found: $405.1820 \mathrm{C}_{24} \mathrm{H}_{24} \mathrm{~N}_{2} \mathrm{O}_{4}$ requires $\left.[\mathrm{M}+\mathrm{H}]^{+}, 405.1810\right) .{ }^{1} \mathrm{H}$ NMR (500 MHz, DMSO- $d_{6}$ ): $\delta$ 7.73-7.57 (1H, m, H6'), 7.48-7.32 (1H, m, H8), 7.28-7.14 (2H, m, $\left.\mathrm{H}^{\prime}, \mathrm{H}^{\prime}\right), 7.12\left(2 \mathrm{H}, \mathrm{d}, J=9.3 \mathrm{~Hz}, \mathrm{H}^{\prime \prime}, \mathrm{H}^{\prime \prime}\right), 7.02-6.85(1 \mathrm{H}, \mathrm{m}$, $\mathrm{H} 7), 6.85(1 \mathrm{H}, \mathrm{d}, J=8.9 \mathrm{~Hz}, \mathrm{H} 5), 6.75(1 \mathrm{H}, \mathrm{dd}, J=8.9,2.7 \mathrm{~Hz}, \mathrm{H} 6)$, 6.15 (1H, s, H7" ), 5.19 (2H, s, H1" ), 3.74-3.45 (12H, m, $4 \times \mathrm{OMe})$. ${ }^{13} \mathrm{C}$ NMR (126 MHz, DMSO- $\left.d_{6}\right): \delta 153.42(\mathrm{C}-2), 153.32\left(\mathrm{C}-5^{\prime}\right)$, $151.90\left(\mathrm{C}-6^{\prime \prime}\right), 151.58\left(\mathrm{C}-3^{\prime \prime}\right), 151.01\left(\mathrm{C}-2^{\prime}\right), 143.22$ (C-4), 135.63 (C9), 125.80 (C-2" $), 122.76$ (C-7), 122.10 (C-6), 120.61 (C-8), 119.56

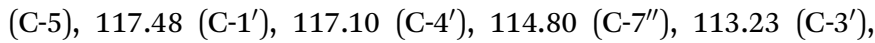
113.00 (C-6'), 112.19 (C-5"), 111.37 (C-4" $), 56.22$ (OMe-5'), 56.04 $\left(\mathrm{OMe}-6^{\prime \prime}\right), 55.68$ (OMe-3"), 55.37 (OMe-2'), 43.26 (C-1").

Method B. Stirring time $=24 \mathrm{~h}$. Purification by washing with cold acetonitrile $(3 \times 20.0 \mathrm{~mL})$ to remove any excess starting material (confirmed by TLC) to afford an oil $(3.64 \mathrm{~g}, 90 \%) . R_{\mathrm{f}}$ (40\% EtOAc/hexane) 0.36 .

Method C. Stirring time $=24 \mathrm{~h}$. Purification by washing with cold acetonitrile $(3 \times 20.0 \mathrm{~mL})$ to remove any excess starting material (confirmed by TLC) to afford an oil $(2.82 \mathrm{~g}, 70 \%) . R_{\mathrm{f}}$ (40\% EtOAc/hexane) 0.36 .

Method D. Stirring time $=24 \mathrm{~h}$. Purification by washing with cold acetonitrile $(3 \times 20.0 \mathrm{~mL})$ to remove any excess starting material (confirmed by TLC) to afford an oil $(3.79 \mathrm{~g}, 94 \%) . R_{\mathrm{f}}$ (40\% EtOAc/hexane) 0.36 .

Synthesis of 1-(3,4-dimethoxybenzyl)-2-(3,4-dimethoxyphenyl)$1 \mathrm{H}$-benzimidazole (4d)

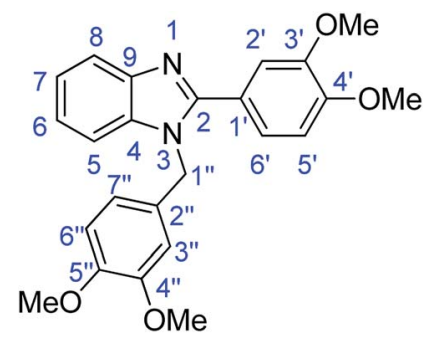

Method A. Stirring time $=24 \mathrm{~h}$. Purification by washing with cold acetonitrile $(3 \times 20.0 \mathrm{~mL})$ to remove any excess starting material (confirmed by TLC) to afford a yellow oil (1.45 g, 36\%).
$R_{\mathrm{f}}(60 \%$ EtOAc/hexane $)$ 0.21. ([M $\left.+\mathrm{H}\right]^{+}$found: 405.1805 $\mathrm{C}_{24} \mathrm{H}_{24} \mathrm{~N}_{2} \mathrm{O}_{4}$ requires $\left.[\mathrm{M}+\mathrm{H}]^{+}, 405.1810\right) .{ }^{1} \mathrm{H}$ NMR $(500 \mathrm{MHz}$, DMSO- $\left.d_{6}\right): \delta 7.88\left(1 \mathrm{H}, \mathrm{s}, \mathrm{H} 2^{\prime}\right), 7.71\left(1 \mathrm{H}, \mathrm{d}, J=7.9 \mathrm{~Hz}, \mathrm{H}^{\prime}\right), 7.53$ (1H, d, $J=7.8 \mathrm{~Hz}, \mathrm{H} 8), 7.34-7.16$ (3H, m, H5, H6, H7), 6.91-6.79 $\left(2 \mathrm{H}, \mathrm{m}, \mathrm{H}^{\prime}, \mathrm{H6}^{\prime \prime}\right), 6.74\left(1 \mathrm{H}, \mathrm{s}, \mathrm{H} 3^{\prime \prime}\right), 6.62(1 \mathrm{H}, \mathrm{d}, J=16.3 \mathrm{~Hz}$, $\left.\mathrm{H}^{\prime \prime}\right), 5.90\left(2 \mathrm{H}, \mathrm{m}, \mathrm{H} 1^{\prime \prime}\right), 3.88-3.57$ (12H, m, $\left.4 \times \mathrm{OMe}\right) .{ }^{13} \mathrm{C} \mathrm{NMR}$ (126 MHz, DMSO- $d_{6}$ ): $\delta 153.71$ (C-2), 150.59 (C-3'), $149.41\left(\mathrm{C}-4^{\prime}\right)$, $149.36\left({\mathrm{C}-4^{\prime \prime}}^{\prime \prime}, 149.18\left(\mathrm{C}-5^{\prime \prime}\right), 148.60\right.$ (C-4), 143.10 (C-9), 129.87 (C$\left.1^{\prime}\right), 122.99$ (C-7), 122.85 (C-6), $122.16\left(\mathrm{C}-2^{\prime \prime}\right), 119.76$ (C-6'), 119.45 (C-7"), 118.95 (C-8), 118.56 (C-5), 112.36 (C-2'), 112.49 (C-6'), $111.37\left({\mathrm{C}-5^{\prime}}^{\prime}\right), 110.78\left(\mathrm{C}-3^{\prime \prime}\right), 56.11$ ( $\left.4 \times \mathrm{OMe}\right), 47.74\left(\mathrm{C}-1^{\prime \prime}\right) . \mathrm{IR}$ $\left(v_{\max } / \mathrm{cm}^{-1}\right): 2970(\operatorname{ArC}-\mathrm{H}) ; 1651(\mathrm{C}=\mathrm{N}) ; 1454(\mathrm{ArC}=\mathrm{C}) ; 1228$ (C-O).

Method B. Stirring time $=24 \mathrm{~h}$. Purification by washing with cold acetonitrile $(3 \times 20.0 \mathrm{~mL})$ to remove any excess starting material (confirmed by TLC) to afford a yellow oil ( $2.66 \mathrm{~g}, 66 \%)$. $R_{\mathrm{f}}$ (60\% EtOAc/hexane) 0.21 .

Method D. Stirring time $=24 \mathrm{~h}$. Purification by washing with cold acetonitrile $(3 \times 20.0 \mathrm{~mL})$ to remove any excess starting material (confirmed by TLC) to afford a white solid $(0.927 \mathrm{~g}$, $23 \%) . R_{\mathrm{f}}(60 \%$ EtOAc/hexane) 0.21 .

Method E. Denilite ${ }^{\circledR}$ II Base $(0.085 \mathrm{~g})$, stirring time $=24 \mathrm{~h}$. Purification by washing with cold acetonitrile $(3 \times 20.0 \mathrm{~mL})$ to remove any excess starting material (confirmed by TLC) to afford a yellow oil $(1.21 \mathrm{~g}, 30 \%) . R_{\mathrm{f}}(60 \%$ EtOAc/hexane) 0.21 .

\section{The following methods were used to synthesize benzothiazoles}

Method F. A mixture of 2-aminothiophenol (1.63 g, 15.0 $\mathrm{mmol})$ and benzaldehyde derivative $(1.06 \mathrm{~g}, 10.0 \mathrm{mmol})$ in acetonitrile $(10.0 \mathrm{~mL})$ and acetate buffer $(10.0 \mathrm{~mL}, \mathrm{pH} 4.0)$ was stirred at room for 5 minutes. Suberase ${ }^{\circledR}(2.0 \mathrm{~mL})$ was then added into the mixture and the contents were stirred for $1 \mathrm{~h}$. When the reaction completes, the product precipitates from the solution and was extracted with ethyl acetate $(30.0 \mathrm{~mL})$ and water $(3 \times 20.0 \mathrm{~mL})$ and concentrated on a rotary evaporator. The product was washed several times with cold acetonitrile (3 $\times 20.0 \mathrm{~mL}$ ) to remove any excess starting material.

Method G. The same as Method A, except that methanol (2.0 $\mathrm{mL}$ ) was used instead of acetonitrile.

Method H. The same as Method A, except that ethanol (4.0 $\mathrm{mL}$ ) was used instead of acetonitrile.

Method I. The same as Method A, except that DMF (10.0 mL) was used instead of acetonitrile.

Method J. The same as Method A, except that DCM $(10.0 \mathrm{~mL})$ was used instead of acetonitrile.

Synthesis of 2-phenylbenzothiazole (7a)

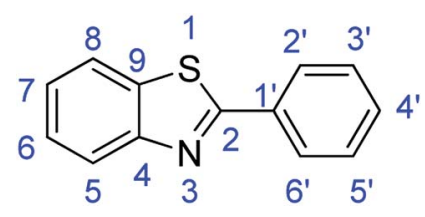


Method F. Stirring time $=2$ h. Purification by washing with cold acetonitrile $(3 \times 20.0 \mathrm{~mL})$ to remove any excess starting material (confirmed by TLC) to afford a bright yellow solid (1.79 g, 85\%). $R_{\mathrm{f}}\left(40 \%\right.$ EtOAc/hexane) $0.93 . \mathrm{Mp}=96-98^{\circ} \mathrm{C}$ (lit. $\left.46102-104{ }^{\circ} \mathrm{C}\right) \cdot\left([\mathrm{M}+\mathrm{H}]^{+}\right.$found: $212.0528 \mathrm{C}_{13} \mathrm{H}_{9} \mathrm{NS}$ requires $[\mathrm{M}$ $\left.+\mathrm{H}]^{+}, 212.0530\right) .{ }^{1} \mathrm{H}$ NMR $(500 \mathrm{MHz}$, chloroform- $d): \delta 8.15-$ $8.04\left(3 \mathrm{H}, \mathrm{m}, \mathrm{H} 8, \mathrm{H} 2^{\prime}, \mathrm{H}^{\prime}\right), 7.91(1 \mathrm{H}, \mathrm{d}, J=8.0 \mathrm{~Hz}, \mathrm{H} 5), 7.50$ $\left(4 \mathrm{H}, \mathrm{m}, \mathrm{H} 6, \mathrm{H} 7, \mathrm{H}^{\prime}, \mathrm{H}^{\prime}\right), 7.39\left(1 \mathrm{H}, \mathrm{t}, J=7.6 \mathrm{~Hz}, \mathrm{H} 4^{\prime}\right) .{ }^{13} \mathrm{C} \mathrm{NMR}$ (126 MHz, chloroform- $d$ ): $\delta 154.17$ (C-2), 135.08 (C-4), 133.65 $\left(\mathrm{C}-1^{\prime}\right), 130.97$ (C-9), 129.03 (C-2', C-6'), 127.57 (C-5'), 126.32 (C$\left.3^{\prime}\right), 125.19$ (C-4'), 123.26 (C-6, C-7), 121.62 (C-5, C-8). IR ( $v_{\max } /$ $\left.\mathrm{cm}^{-1}\right): 3075(\mathrm{ArC}-\mathrm{H}) ; 1588,1558(\mathrm{ArC}=\mathrm{C}) ; 1644(\mathrm{C}=\mathrm{N}) ; 727$ (C-S).

Method G. Stirring time $=24 \mathrm{~h}$. Purification by washing with cold acetonitrile $(3 \times 20.0 \mathrm{~mL})$ to remove any excess starting material (confirmed by TLC) to afford a white solid $(1.23 \mathrm{~g}, 58 \%) . R_{\mathrm{f}}$ (40\% EtOAc/hexane) 0.93 .

Method H. Stirring time $=24 \mathrm{~h}$. Purification by washing with cold acetonitrile $(3 \times 20.0 \mathrm{~mL})$ to remove any excess starting material (confirmed by TLC) to afford a white solid (1.16 g, $55 \%) . R_{\mathrm{f}}$ (40\% EtOAc/hexane) 0.93 .

Method I. Stirring time $=24 \mathrm{~h}$. Purification by washing with cold acetonitrile $(3 \times 20.0 \mathrm{~mL})$ to remove any excess starting material (confirmed by TLC) to afford a white solid (1.66 g, $78 \%) . R_{\mathrm{f}}(40 \%$ EtOAc/hexane) 0.93 .

Method J. Stirring time $=24 \mathrm{~h}$. Purification by washing with cold acetonitrile $(3 \times 20.0 \mathrm{~mL})$ to remove any excess starting material (confirmed by TLC) to afford a white solid (1.37 g, $65 \%) . R_{\mathrm{f}}(40 \%$ EtOAc/hexane) 0.93 .

\section{Synthesis of 4-(benzothiazol-2-yl)-N,N-dimethylaniline (7b)}

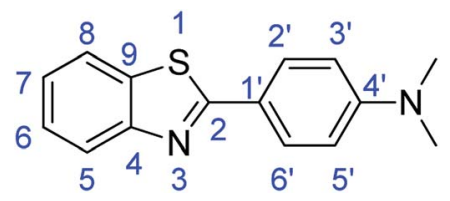

Method F. Stirring time $=2 \mathrm{~h}$. Purification by washing with cold acetonitrile $(3 \times 20.0 \mathrm{~mL})$ to remove any excess starting material (confirmed by TLC) to afford a cream solid (1.43 g, $56 \%$ ). $R_{\mathrm{f}}\left(40 \%\right.$ EtOAc/hexane) $0.81 . \mathrm{Mp}=172{ }^{\circ} \mathrm{C}$ (lit. $47170-$ $\left.171{ }^{\circ} \mathrm{C}\right) .\left([\mathrm{M}+\mathrm{H}]^{+}\right.$found: $255.0984 \mathrm{C}_{15} \mathrm{H}_{14} \mathrm{~N}_{2} \mathrm{~S}$ requires $[\mathrm{M}+$ $\left.\mathrm{H}]^{+}, 255.0952\right) .{ }^{1} \mathrm{H}$ NMR (500 MHz, chloroform- $d$ ): $\delta 8.03-7.91$ $\left(2 \mathrm{H}, \mathrm{m}, \mathrm{H}^{\prime}, \mathrm{H6}^{\prime}\right), 7.84(1 \mathrm{H}, \mathrm{d}, J=7.9 \mathrm{~Hz}, \mathrm{H} 5), 7.44(1 \mathrm{H}, \mathrm{t}, J=$ $7.4 \mathrm{~Hz}, \mathrm{H6}), 7.30(1 \mathrm{H}, \mathrm{t}, J=7.6 \mathrm{~Hz}, \mathrm{H} 7), 6.82-6.54(3 \mathrm{H}, \mathrm{m}, \mathrm{H} 8$, $\left.\mathrm{H}^{\prime}, \mathrm{H}^{\prime}\right), 3.01\left(6 \mathrm{H}, \mathrm{d}, J=53.2 \mathrm{~Hz}, \mathrm{~N}-\mathrm{CH}_{3}\right) .{ }^{13} \mathrm{C} \mathrm{NMR}(126 \mathrm{MHz}$, chloroform- $d$ ): $\delta 168.79$ (C-2), 154.43 (C-4'), 152.20 (C-4), 134.56 (C-9), 128.87 (C-6'), 127.71 (C-2'), 125.97 (C-6), 125.29 (C-7), $124.18\left(\mathrm{C}-1^{\prime}\right), 122.29(\mathrm{C}-8), 121.34$ (C-5), $120.46\left(\mathrm{C}-5^{\prime}\right)$, 112.29(C-3'), $40.48\left(\mathrm{~N}-\mathrm{CH}_{3}\right), 40.18\left(\mathrm{~N}-\mathrm{CH}_{3}\right)$. IR $\left(v_{\max } / \mathrm{cm}^{-1}\right)$ : $3053($ ArC-H); $2904(\mathrm{C}-\mathrm{N}) ; 2815(\mathrm{~N}-\mathrm{C}-\mathrm{H}), 1556,1526(\mathrm{ArC}=\mathrm{C})$; $1604(\mathrm{C}=\mathrm{N}) ; 720(\mathrm{C}-\mathrm{S})$.

\section{Synthesis of 2-(4-methoxyphenyl)benzothiazole (7c)}

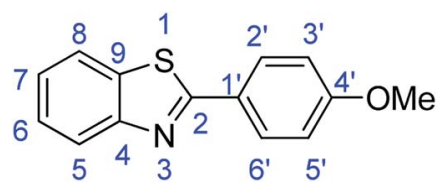

Method F. Stirring time $=8 \mathrm{~h}$. Purification by washing with cold acetonitrile $(3 \times 20.0 \mathrm{~mL})$ to remove any excess starting material (confirmed by TLC) to afford white solid (1.64 g, 68\%). $R_{\mathrm{f}}$ (40\% EtOAc/hexane) 0.89. $\mathrm{Mp}=120-122{ }^{\circ} \mathrm{C}$ (lit. $48120-122{ }^{\circ} \mathrm{C}$ ). $\left([\mathrm{M}+\mathrm{H}]^{+}\right.$found: $242.0632 \mathrm{C}_{14} \mathrm{H}_{11}$ NOS requires $\left.[\mathrm{M}+\mathrm{H}]^{+}, 242.0635\right)$. ${ }^{1} \mathrm{H}$ NMR (500 MHz, chloroform- $d$ ): $\delta 8.04(3 \mathrm{H}, \mathrm{d}, J=8.7 \mathrm{~Hz}, \mathrm{H} 5$, $\left.\mathrm{H} 2^{\prime}, \mathrm{H6}^{\prime}\right), 7.88(1 \mathrm{H}, \mathrm{d}, J=8.0 \mathrm{~Hz}, \mathrm{H} 8), 7.47(1 \mathrm{H}, \mathrm{t}, J=7.6 \mathrm{~Hz}, \mathrm{H} 6)$, $7.35(1 \mathrm{H}, \mathrm{t}, J=7.6 \mathrm{~Hz}, \mathrm{H} 7), 7.00\left(2 \mathrm{H}, \mathrm{d}, J=8.7 \mathrm{~Hz}, \mathrm{H}^{\prime}, \mathrm{H}^{\prime}\right), 3.88$ $(3 \mathrm{H}, \mathrm{s}, \mathrm{OMe}) \cdot{ }^{13} \mathrm{C}$ NMR (126 MHz, chloroform- $d$ ): $\delta 167.85$ (C-2), 161.94 (C-4'), 154.24 (C-4), 134.87 (C-1'), 129.12 (C-9), 126.47 (C6'), 126.19 (C-2'), 124.79 (C-6), 122.83 (C-7), 121.50 (C-5, C-8), 114.38 $\left(\mathrm{C}-3^{\prime}, \mathrm{C}^{\prime} 5^{\prime}\right), 55.46$ (OMe). IR $\left(v_{\max } / \mathrm{cm}^{-1}\right): 3060$ (ArC-H); 1574, 1557 $(\mathrm{ArC}=\mathrm{C}) ; 1603(\mathrm{C}=\mathrm{N}) ; 729(\mathrm{C}-\mathrm{S}), 1310(\mathrm{C}-\mathrm{O})$.

\section{Synthesis of 2-(3,4-dimethoxyphenyl)benzothiazole (7d)}

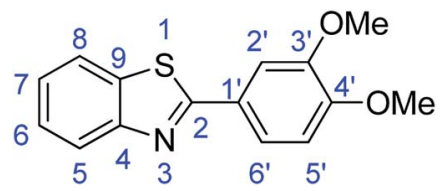

Method F. Stirring time $=2$ h. Purification by washing with cold acetonitrile $(3 \times 20.0 \mathrm{~mL})$ to remove any excess starting material (confirmed by TLC) to afford a cream solid (1.98 g, $73 \%) . R_{\mathrm{f}}\left(40 \%\right.$ EtOAc/hexane) 0.67. $\mathrm{Mp}=132-134{ }^{\circ} \mathrm{C}$ (lit. 48 $\left.132-134{ }^{\circ} \mathrm{C}\right) \cdot\left([\mathrm{M}+\mathrm{H}]^{+}\right.$found: $272.0740 \mathrm{C}_{15} \mathrm{H}_{13} \mathrm{NO}_{2} \mathrm{~S}$ requires $[\mathrm{M}$ $\left.+\mathrm{H}]^{+}, 272.0741\right) .{ }^{1} \mathrm{H}$ NMR $(500 \mathrm{MHz}$, chloroform- $d): \delta 8.04(1 \mathrm{H}$, $\mathrm{d}, J=8.2 \mathrm{~Hz}, \mathrm{H} 5), 7.88(1 \mathrm{H}, \mathrm{d}, J=7.9 \mathrm{~Hz}, \mathrm{H} 8), 7.71(1 \mathrm{H}, \mathrm{s}, \mathrm{H} 2)$, $7.60\left(1 \mathrm{H}, \mathrm{d}, J=9.5 \mathrm{~Hz}, \mathrm{H}^{\prime}\right), 7.47(1 \mathrm{H}, \mathrm{t}, J=7.7 \mathrm{~Hz}, \mathrm{H} 6), 7.36$ $(1 \mathrm{H}, \mathrm{t}, J=7.6 \mathrm{~Hz}, \mathrm{H} 7), 6.95\left(1 \mathrm{H}, \mathrm{d}, J=8.4 \mathrm{~Hz}, \mathrm{H}^{\prime}\right), 4.03(3 \mathrm{H}, \mathrm{s}$, OMe), 3.96 (3H, s, OMe). ${ }^{13} \mathrm{C}$ NMR (126 MHz, chloroform- $d$ ): $\delta 167.93$ (C-2), 154.17 (C-4), $151.60\left(\mathrm{C}^{\prime} 3^{\prime}\right), 149.37\left(\mathrm{C}-4^{\prime}\right), 134.92$ (C-9), 126.71 (C-1'), 126.23 (C-6), 124.88 (C-7), 122.85 (C-5), 121.51 (C-8), 121.16 (C-6'), 111.05 (C-2'), $109.84\left(\mathrm{C}-5^{\prime}\right), 56.15$ $\left(\mathrm{OMe}-3^{\prime}\right), 56.06$ (OMe-4'). IR $\left(v_{\text {max }} / \mathrm{cm}^{-1}\right): 2964(\mathrm{ArC}-\mathrm{H}) ; 1520$, $1479(\operatorname{ArC}=\mathrm{C}) ; 1598(\mathrm{C}=\mathrm{N}) ; 732(\mathrm{C}-\mathrm{S}), 1312(\mathrm{C}-\mathrm{O})$.

\section{Synthesis of 2-(3,4,5-trimethoxyphenyl)benzothiazole (7e)}

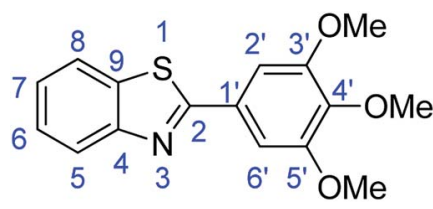


Method F. Stirring time $=2 \mathrm{~h}$. Purification by washing with cold acetonitrile $(3 \times 20.0 \mathrm{~mL})$ to remove any excess starting material (confirmed by TLC) to afford a yellow solid (1.96 g, $65 \%) . R_{\mathrm{f}}$ (40\% EtOAc/hexane) 0.74. $\mathrm{Mp}=148-151{ }^{\circ} \mathrm{C}$ (lit. 49 149.5-151.1 $\left.{ }^{\circ} \mathrm{C}\right)\left([\mathrm{M}+\mathrm{H}]^{+}\right.$found: $302.0836 \mathrm{C}_{16} \mathrm{H}_{15} \mathrm{NO}_{3} \mathrm{~S}$ requires $\left.[\mathrm{M}+\mathrm{H}]^{+}, 302.0847\right) .{ }^{1} \mathrm{H}$ NMR (500 MHz, chloroform- $d$ ): $\delta 8.06$ $(1 \mathrm{H}, \mathrm{d}, J=8.2 \mathrm{~Hz}, \mathrm{H} 5), 7.89(1 \mathrm{H}, \mathrm{d}, J=8.0 \mathrm{~Hz}, \mathrm{H} 8), 7.49(1 \mathrm{H}, \mathrm{t}, J$ $=7.7 \mathrm{~Hz}, \mathrm{H} 6), 7.38(1 \mathrm{H}, \mathrm{t}, J=7.6 \mathrm{~Hz}, \mathrm{H} 7), 7.34\left(2 \mathrm{H}, \mathrm{s}, \mathrm{H} 2^{\prime}, \mathrm{H}^{\prime}\right)$, $3.96(9 \mathrm{H}, \mathrm{d}, J=30.7 \mathrm{~Hz}, \mathrm{OMe}) .{ }^{13} \mathrm{C}$ NMR $(126 \mathrm{MHz}$, Chloroformd): $\delta 167.80$ (C-2), 154.10 (C-4), $153.61\left(\mathrm{C}-3^{\prime}, \mathrm{C}-5^{\prime}\right), 140.71\left(\mathrm{C}-4^{\prime}\right)$, 135.06 (C-9), 129.09 (C-1'), 126.36 (C-6), 125.13 (C-7), 123.10 (C8), 121.56 (C-5), 104.84 (C-2', C-6'), 61.02 ( $2 \times$ OMe), 56.39 (OMe). IR $\left(v_{\max } / \mathrm{cm}^{-1}\right): 2941(\mathrm{ArC}-\mathrm{H}) ; 1581,1518(\mathrm{ArC}=\mathrm{C}) ; 1698$ $(\mathrm{C}=\mathrm{N}) ; 1331(\mathrm{C}-\mathrm{O}) ; 709(\mathrm{C}-\mathrm{S})$.

\section{Synthesis of 2-(2-chlorophenyl)benzothiazole (7f)}

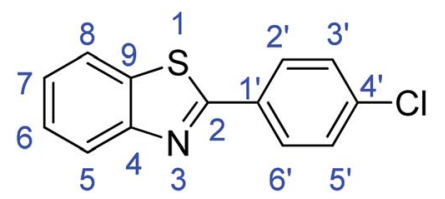

Method F. Stirring time $=18 \mathrm{~h}$. Purification by washing with cold acetonitrile $(3 \times 20.0 \mathrm{~mL})$ to remove any excess starting material (confirmed by TLC) to afford a cream solid (1.35 g, $55 \%) . R_{\mathrm{f}}\left(40 \%\right.$ EtOAc/hexane) 0.93. $\mathrm{Mp}=110-112{ }^{\circ} \mathrm{C}$ (lit. 50 110-112 $\left.{ }^{\circ} \mathrm{C}\right) \cdot\left([\mathrm{M}+\mathrm{H}]^{+}\right.$found: $246.0139 \mathrm{C}_{13} \mathrm{H}_{8} \mathrm{ClNS}$ requires $[\mathrm{M}+$ $\left.\mathrm{H}]^{+}, 246.0140\right) .{ }^{1} \mathrm{H}$ NMR (500 MHz, chloroform- $d$ ) $\delta 8.07(1 \mathrm{H}, \mathrm{d}, J$ $=8.2 \mathrm{~Hz}, \mathrm{H} 5), 8.03\left(2 \mathrm{H}, \mathrm{d}, J=8.4 \mathrm{~Hz}, \mathrm{H}^{\prime}, \mathrm{H6}^{\prime}\right), 7.90(1 \mathrm{H}, \mathrm{d}, J=$ $8.0 \mathrm{~Hz}, \mathrm{H} 8), 7.48$ (3H, m, H6, H3 $\left.{ }^{\prime}, \mathrm{H}^{\prime}\right), 7.40(1 \mathrm{H}, \mathrm{t}, J=7.6 \mathrm{~Hz}$, $\mathrm{H} 7) .{ }^{13} \mathrm{C}$ NMR (126 MHz, chloroform- $d$ ) $\delta 166.60$ (C-2), 154.08 (C-

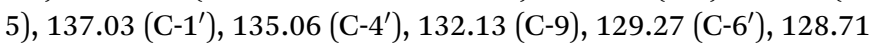
$\left(\mathrm{C}-2^{\prime}\right), 126.48$ (C-3' $\left.{ }^{\prime}, \mathrm{C}^{\prime} 5^{\prime}\right), 125.41$ (C-6), 123.31 (C-7), 121.65 (C-5, C-8). IR $\left(v_{\max } / \mathrm{cm}^{-1}\right): 3055(\mathrm{ArC}-\mathrm{H}) ; 1646(\mathrm{C}=\mathrm{N}) ; 1588,1569$ $(\mathrm{ArC}=\mathrm{C}) ; 730(\mathrm{C}-\mathrm{Cl}), 709(\mathrm{C}-\mathrm{S})$.

\section{Synthesis of 2-(pyridin-4-yl)benzothiazole (7g)}

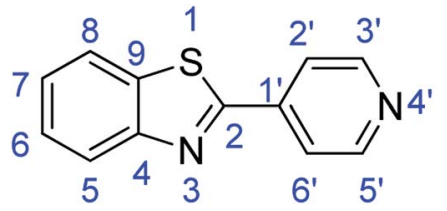

Method F. Stirring time $=2$ h. Purification by washing with cold acetonitrile $(3 \times 20.0 \mathrm{~mL})$ to remove any excess starting material (confirmed by TLC) to afford a bright yellow solid $(1.86 \mathrm{~g}, 88 \%) . R_{\mathrm{f}}\left(40 \%\right.$ EtOAc/hexane) $0.37 . \mathrm{Mp}=126-130{ }^{\circ} \mathrm{C}$ (lit. $\left.51126-130{ }^{\circ} \mathrm{C}\right) \cdot\left([\mathrm{M}+\mathrm{H}]^{+}\right.$found: $213.0481 \mathrm{C}_{12} \mathrm{H}_{8} \mathrm{~N}_{2} \mathrm{~S}$ requires [M $\left.+\mathrm{H}]^{+}, 213.0482\right) .{ }^{1} \mathrm{H}$ NMR $(500 \mathrm{MHz}$, chloroform- $d): \delta 8.78(2 \mathrm{H}$, $\left.\mathrm{d}, J=5.7 \mathrm{~Hz}, \mathrm{H3}^{\prime}, \mathrm{H}^{\prime}\right), 8.13(1 \mathrm{H}, \mathrm{d}, J=8.2 \mathrm{~Hz}, \mathrm{H} 5), 7.99-7.87$ $\left(3 \mathrm{H}, \mathrm{m}, \mathrm{H} 8, \mathrm{H}^{\prime}, \mathrm{H}^{\prime}\right), 7.55(1 \mathrm{H}, \mathrm{t}, J=7.7 \mathrm{~Hz}, \mathrm{H} 6), 7.46(1 \mathrm{H}, \mathrm{t}, J=$ $7.6 \mathrm{~Hz}, \mathrm{H} 7) .{ }^{13} \mathrm{C}$ NMR (126 MHz, chloroform- $d$ ): $\delta 165.10$ (C-4), 153.99 (C-2), 150.77 (C-3', C-5'), 140.49 (C-1'), 135.23 (C-9),
126.82 (C-6), 126.20 (C-7), 123.94 (C-8), 121.87 (C-5), 121.20 (C$\left.2^{\prime}, \mathrm{C}-6^{\prime}\right)$. IR ( $\left.v_{\max } / \mathrm{cm}^{-1}\right): 3035(\mathrm{ArC}-\mathrm{H}) ; 1698(\mathrm{C}=\mathrm{N}) ; 1552,1503$ $(\mathrm{ArC}=\mathrm{C}) ; 704(\mathrm{C}-\mathrm{S})$.

Synthesis of 1,4-bis(benzothiazol-2-yl)benzene (7h)

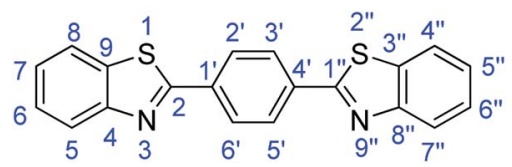

Method F. 2-Aminothiophenol (2 equiv.), stirring time $=2 \mathrm{~h}$. Purification by washing with cold acetonitrile $(3 \times 20.0 \mathrm{~mL})$ to remove any excess starting material (confirmed by TLC) to afford a yellow solid (2.98 g, 87\%). $R_{\mathrm{f}}(40 \%$ EtOAc/hexane) 0.81 . $\mathrm{Mp}=261-264{ }^{\circ} \mathrm{C}$ (lit. $\left.52263{ }^{\circ} \mathrm{C}\right) .\left([\mathrm{M}+\mathrm{H}]^{+}\right.$found: 345.0515 $\mathrm{C}_{20} \mathrm{H}_{12} \mathrm{~N}_{2} \mathrm{~S}_{2}$ requires $\left.[\mathrm{M}+\mathrm{H}]^{+}, 345.0516\right) .{ }^{1} \mathrm{H}$ NMR $(500 \mathrm{MHz}$, chloroform- $d$ ): $\delta 8.23$ (4H, s, H2 $\left.{ }^{\prime}, \mathrm{H}^{\prime}, \mathrm{H}^{\prime}, \mathrm{H}^{\prime}\right), 8.12(2 \mathrm{H}, \mathrm{d}, J=$ $\left.8.1 \mathrm{~Hz}, \mathrm{H} 5, \mathrm{H}^{\prime \prime}\right), 7.94\left(2 \mathrm{H}, \mathrm{d}, J=8.0 \mathrm{~Hz}, \mathrm{H} 8, \mathrm{H}^{\prime \prime}\right), 7.53(2 \mathrm{H}, \mathrm{t}, J$ $\left.=7.6 \mathrm{~Hz}, \mathrm{H6}, \mathrm{H6}^{\prime \prime}\right), 7.42\left(2 \mathrm{H}, \mathrm{t}, J=7.5 \mathrm{~Hz}, \mathrm{H} 7, \mathrm{H} 5^{\prime \prime}\right) .{ }^{13} \mathrm{C} \mathrm{NMR}$ (126 MHz, chloroform- $d$ ): $\delta 166.81\left(\mathrm{C}-2, \mathrm{C}-1^{\prime \prime}\right), 154.19$ (C-4, C-8" ), $135.70\left(\mathrm{C}-1^{\prime}, \mathrm{C}-4^{\prime \prime}\right), 135.21$ (C-9, C-3"), 128.09 (C-2' $, \mathrm{C}-3^{\prime}, \mathrm{C}-5^{\prime}, \mathrm{C}-$ 6'), 126.56 (C-6, C-6"), 125.56 (C-7, C-5"), 123.48 (C-8, C-4"), $121.70\left(\mathrm{C}-5, \mathrm{C}-7^{\prime \prime}\right)$. IR $\left(v_{\max } / \mathrm{cm}^{-1}\right): 3057(\mathrm{ArC}-\mathrm{H}) ; 1697(\mathrm{C}=\mathrm{N})$; 1558, $1522(\mathrm{ArC}=\mathrm{C}) ; 724(\mathrm{C}-\mathrm{S})$.

\section{Synthesis of 2-(2-bromo-5-methoxyphenyl)benzothiazole (7i)}

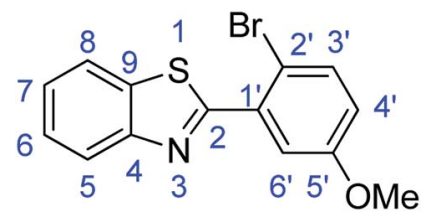

Method F. Stirring time $=8 \mathrm{~h}$. Purification by washing with cold acetonitrile $(3 \times 20.0 \mathrm{~mL})$ to remove any excess starting material (confirmed by TLC) to afford a yellow solid $(2.42 \mathrm{~g}$, $76 \%) . R_{\mathrm{f}}$ (40\% EtOAc/hexane) 0.93. $\mathrm{Mp}=123-125{ }^{\circ} \mathrm{C}$. $([\mathrm{M}+$ $\mathrm{H}]^{+}$found: $319.9739 \mathrm{C}_{14} \mathrm{H}_{10}$ BrNOS requires $[\mathrm{M}+\mathrm{H}]^{+}$, 319.9740). ${ }^{1} \mathrm{H}$ NMR (500 MHz, chloroform- $d$ ): $\delta 8.68(1 \mathrm{H}, \mathrm{d}, J$ $=2.3 \mathrm{~Hz}, \mathrm{H} 5), 8.10(1 \mathrm{H}, \mathrm{d}, J=8.2 \mathrm{~Hz}, \mathrm{H} 8), 7.93(1 \mathrm{H} \mathrm{d}, J=$ $\left.7.9 \mathrm{~Hz}, \mathrm{H3}^{\prime}\right), 7.57-7.46\left(2 \mathrm{H}, \mathrm{m}, \mathrm{H} 6, \mathrm{H6}^{\prime}\right), 7.39(1 \mathrm{H}, \mathrm{t}, J=$ $7.5 \mathrm{~Hz}, \mathrm{H} 7), 6.94$ (1H, d, $\left.J=8.8 \mathrm{~Hz}, \mathrm{H} 4^{\prime}\right), 4.04$ (3H, s, OMe). ${ }^{13} \mathrm{C}$ NMR (126 MHz, chloroform- $d$ ): $\delta 161.43(\mathrm{C}-2), 156.22(\mathrm{C}-$ 5'), 152.00 (C-4), 136.20 (C-1'), 134.12 (C-9), 131.88 (C-3'), 126.11 (C-6), 124.93 (C-7), 124.05 (C-8), 122.99 (C-5), 121.24 (C-4'), 113.81 (C-6'), $113.46\left(\mathrm{C}-2^{\prime}\right), 56.03$ (OMe). IR ( $v_{\max } /$ $\left.\mathrm{cm}^{-1}\right)$ : $3058(\operatorname{ArC}-\mathrm{H}) ; 1694(\mathrm{C}=\mathrm{N}) ; 1587,1571(\mathrm{ArC}=\mathrm{C}) ; 1315$ (C-O); 751 (C-Br); 723 (C-S). 
Synthesis of 2-(3-nitrophenyl)benzothiazole (7j)

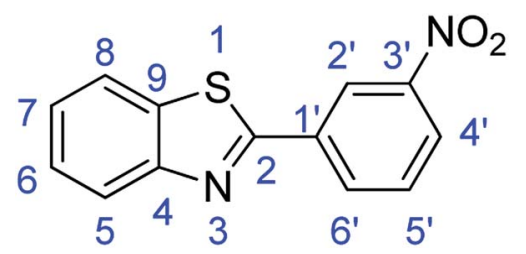

Method F. Stirring time $=8 \mathrm{~h}$. Purification by washing with cold acetonitrile $(3 \times 20.0 \mathrm{~mL})$ to remove any excess starting material (confirmed by TLC) to afford a yellow solid $(1.23 \mathrm{~g}$, $48 \%$ ). $R_{\mathrm{f}}\left(40 \%\right.$ EtOAc/hexane) $0.81 . \mathrm{Mp}=185-187{ }^{\circ} \mathrm{C}$ (lit. 53 186.8-187.3 $\left.{ }^{\circ} \mathrm{C}\right)$. $\left([\mathrm{M}+\mathrm{H}]^{+}\right.$found: $257.0379 \mathrm{C}_{13} \mathrm{H}_{8} \mathrm{~N}_{2} \mathrm{O}_{2} \mathrm{~S}$ requires $\left.[\mathrm{M}+\mathrm{H}]^{+}, 257.0380\right) .{ }^{1} \mathrm{H}$ NMR $(500 \mathrm{MHz}$, chloroformd): $\delta 8.94\left(1 \mathrm{H}, \mathrm{s}, \mathrm{H}^{\prime}\right), 8.43\left(1 \mathrm{H}, \mathrm{d}, J=7.8 \mathrm{~Hz}, \mathrm{H}^{\prime}\right), 8.34(1 \mathrm{H}, \mathrm{d}, J$ $\left.=8.2 \mathrm{~Hz}, \mathrm{H} 4^{\prime}\right), 8.12(1 \mathrm{H}, \mathrm{d}, J=8.2 \mathrm{~Hz}, \mathrm{H} 5), 7.95(1 \mathrm{H}, \mathrm{d}, J=$ $8.0 \mathrm{~Hz}, \mathrm{H} 8), 7.69\left(1 \mathrm{H}, \mathrm{t}, J=8.0 \mathrm{~Hz}, \mathrm{H}^{\prime}\right), 7.55(1 \mathrm{H}, \mathrm{t}, J=7.7 \mathrm{~Hz}$, H6), $7.46(1 \mathrm{H}, \mathrm{t}, J=7.6 \mathrm{~Hz}, \mathrm{H} 7) .{ }^{13} \mathrm{C}$ NMR $(126 \mathrm{MHz}$, chloroform- $d$ ): $\delta 164.89$ (C-2), 153.97 (C-4), $148.79\left(\mathrm{C}-3^{\prime}\right), 135.32$ $\left(\mathrm{C}-6^{\prime}\right), 135.20\left(\mathrm{C}-1^{\prime}\right), 132.99$ (C-9), 130.10 (C-5'), 126.84 (C-6), 126.03 (C-7), 125.18 (C-4'), 123.77 (C-2'), 122.35 (C-8), 121.83 (C-5). IR ( $\left.v_{\text {max }} / \mathrm{cm}^{-1}\right)$ : $3085(\operatorname{ArC}-\mathrm{H}) ; 1612(\mathrm{C}=\mathrm{N}) ; 1579,1547$ $(\mathrm{ArC}=\mathrm{C}) ; 1362(\mathrm{~N}-\mathrm{O}) ; 715(\mathrm{C}-\mathrm{S})$.

Synthesis of 2-(2-methoxyphenyl)benzothiazole (7k)

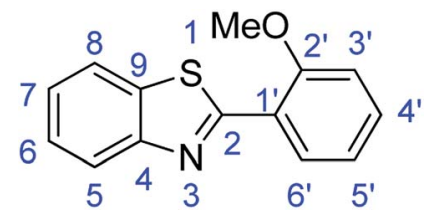

Method F. Stirring time $=24 \mathrm{~h}$. Purification by washing with cold acetonitrile $(3 \times 20.0 \mathrm{~mL})$ to remove any excess starting material (confirmed by TLC) to afford a brown solid $(1.78 \mathrm{~g}, 74 \%) . R_{\mathrm{f}}\left(40 \%\right.$ EtOAc/hexane) $0.89 . \mathrm{Mp}=121{ }^{\circ} \mathrm{C}$ (lit. 54 $\left.120-122{ }^{\circ} \mathrm{C}\right) \cdot\left([\mathrm{M}+\mathrm{H}]^{+}\right.$found: $242.0634 \mathrm{C}_{14} \mathrm{H}_{11}$ NOS requires [M $\left.+\mathrm{H}]^{+}, 242.0635\right) .{ }^{1} \mathrm{H}$ NMR $(500 \mathrm{MHz}$, chloroform- $d): \delta 8.54(1 \mathrm{H}$, $\mathrm{d}, J=7.8 \mathrm{~Hz}, \mathrm{H} 5), 8.10(1 \mathrm{H}, \mathrm{d}, J=8.2 \mathrm{~Hz}, \mathrm{H} 8), 7.93(1 \mathrm{H}, \mathrm{d}, J=$ $\left.7.9 \mathrm{~Hz}, \mathrm{H6}^{\prime}\right), 7.56-7.42(2 \mathrm{H}, \mathrm{m}, \mathrm{H} 6, \mathrm{H} 7), 7.37(1 \mathrm{H}, \mathrm{t}, J=7.5 \mathrm{~Hz}$, $\left.\mathrm{H}^{\prime}\right), 7.14\left(1 \mathrm{H}, \mathrm{t}, J=7.6 \mathrm{~Hz}, \mathrm{H} 4^{\prime}\right), 7.06\left(1 \mathrm{H}, \mathrm{d}, J=8.3 \mathrm{~Hz}, \mathrm{H}^{\prime}\right)$, 4.05 (3H, s, OMe). ${ }^{13} \mathrm{C}$ NMR (126 MHz, chloroform- $d$ ): $\delta 153.49$ (C-2), 150.51 (C-2'), 148.80 (C-4), 137.60 (C-9), 134.06 (C-4'), 133.86 (C-6'), 130.78 (C-6), 129.93 (C-7), 129.60 (C-1'), 125.66

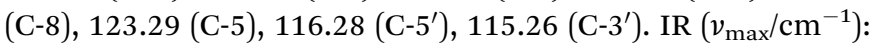
$3053($ ArC-H); $1604(\mathrm{C}=\mathrm{N}) ; 1556,1526(\mathrm{ArC}=\mathrm{C}) ; 1315(\mathrm{C}-\mathrm{O})$; $720(\mathrm{C}-\mathrm{S})$.
Synthesis of 2-(4-nitrophenyl)benzothiazole (7l)

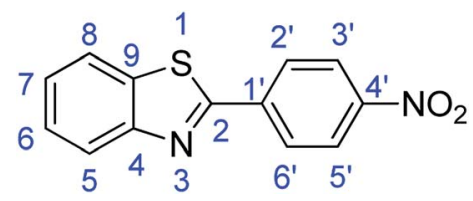

Method F. Stirring time $=24 \mathrm{~h}$. Purification by washing with cold acetonitrile $(3 \times 20.0 \mathrm{~mL})$ to remove any excess starting material (confirmed by TLC) to afford a yellow solid $(2.15 \mathrm{~g}$, $84 \%) . R_{\mathrm{f}}\left(40 \%\right.$ EtOAc/hexane) 0.92. $\mathrm{Mp}=243{ }^{\circ} \mathrm{C}$ (lit. $54233{ }^{\circ} \mathrm{C}$ ). $\left([\mathrm{M}+\mathrm{H}]^{+}\right.$found: $257.0379 \mathrm{C}_{13} \mathrm{H}_{8} \mathrm{~N}_{2} \mathrm{O}_{2} \mathrm{~S}$ requires $[\mathrm{M}+\mathrm{H}]^{+}$, 257.0380). ${ }^{1} \mathrm{H}$ NMR (500 MHz, chloroform- $d$ ): $\delta 8.36(2 \mathrm{H}, \mathrm{d}, J=$ $8.7 \mathrm{~Hz}, \mathrm{H}^{\prime}, \mathrm{H}^{\prime}$ ), 8.27 (2H, d, $\left.J=8.7 \mathrm{~Hz}, \mathrm{H} 2^{\prime}, \mathrm{H}^{\prime}\right), 8.13(1 \mathrm{H}, \mathrm{d}, J$ $=8.2 \mathrm{~Hz}, \mathrm{H} 5), 7.96(1 \mathrm{H}, \mathrm{d}, J=8.0 \mathrm{~Hz}, \mathrm{H} 8), 7.56(1 \mathrm{H}, \mathrm{t}, J=7.7 \mathrm{~Hz}$, H6), 7.47 (1H, t, $J=7.6 \mathrm{~Hz}, \mathrm{H} 7) .{ }^{13} \mathrm{C}$ NMR (126 MHz, chloroform- $d$ ): $\delta 164.84$ (C-2), 154.11 (C-4), 149.05 (C-1'), $139.19\left(\mathrm{C}-4^{\prime}\right)$, 135.50 (C-9), 128.24 (C-2', C-6'), 126.93 (C-6), 126.23 (C-7), 124.32 $\left(\mathrm{C}-3^{\prime}\right), 123.95\left({\mathrm{C}-5^{\prime}}^{\prime}\right), 121.85(\mathrm{C}-5, \mathrm{C}-8)$. IR $\left(v_{\max } / \mathrm{cm}^{-1}\right): 3060$ (ArC$\mathrm{H}) ; 1603(\mathrm{C}=\mathrm{N}) ; 1574,1557(\mathrm{ArC}=\mathrm{C}) ; 1310(\mathrm{~N}-\mathrm{O}) ; 729(\mathrm{C}-\mathrm{S})$.

\section{Conclusions}

Laccases are proving themselves to be very useful oxidising catalysts that enable access to a wide range of chemicals, as was demonstrated here, synthesising pharmaceutically relevant compounds such as 2-aryl- $1 H$-benzimidazoles and 2-arylbenzothiazole derivatives in good to excellent yields. This could be achieved using readily available commercial laccases, making this technique accessible to the general chemistry community.

Chemoselectivity was achieved in the synthesis of 2-aryl- $1 \mathrm{H}^{-}$ benzimidazoles by changing the solvent and laccase to Novoprime Base 268 in an acetate buffer - acetonitrile solvent. The same protocol was applied to the synthesis of 2-arylbenzothiazoles and good to excellent yields were again obtained.

The reaction proceed under very mild conditions, with low environmental impact (green), and the transformations require a simple work-up routine to obtain pure product with a simple wash with cold acetonitrile. In addition, this method eliminated the use of transition metal catalysts (e.g. palladium, ruthenium or copper); use of toxic oxidants such as DDQ, $\mathrm{NiO}_{2}$ or $\mathrm{KI}$ and the release of iodinated intermediates from oxidants; and the use of solvents with major environmental concerns such as DMF.

\section{Conflicts of interest}

There are no conflicts to declare.

\section{Acknowledgements}

We would like to thank Eric Morifi, Thapelo Mmbhele and Refilwe Moepya for technical assistance with Mass Spectrometry and Dr Hendrik Henning for technical assistance with NMR spectrometry: Support from the Department of Science and Technology Biocatalysis Initiative (Grant 0175/2013) is greatly appreciated. 


\section{References}

1 S. Witayakran and A. J. Ragauskas, Adv. Synth. Catal., 2009, 351, 1187-1209.

$2 \mathrm{~K}$. W. Wellington, Application of laccases in organic synthesis: a review, Green Chemistry, Nova Science Publishers, 2012, pp. 167-212, ISBN: 978-161324877-5; K. W. Wellington, R. Bokako, N. Raseroka and P. Steenkamp, Green Chem., 2012, 14, 2567-2576.

3 V. Madhavi and S. Lele, BioRes, 2009, 4, 1694-1717.

4 M.-A. Constantin, J. Conrad and U. Beifuss, Green Chem., 2012, 14, 2375-2379.

5 J. G. Buchanan and H. Z. Sable, in Selective Organic Transformations, ed. B. S. TChyagarajan, Wiley-Interscience, New York, 1972, vol. 2, pp. 1-95.

6 D. M. Reddy and V. K. Reddy, World J. Pharm. Pharm. Sci., 2015, 5, 983-996.

7 H. Leutbecher, M.-A. Constantin, S. Mika, J. Conrad and U. Beifuss, Tetrahedron Lett., 2011, 52, 605-608.

8 V. S. Padalkar, V. D. Gupta, K. R. Phatangare, V. S. Patil, P. G. Umape and N. Sekar, Green Chem. Lett. Rev., 2012, 5, 139-145.

9 J. C. Sih, W. bin Im, A. Robert, D. R. Graber and D. P. Blakeman, J. Med. Chem., 1991, 34, 1049-1062.

10 M. Alamgir, D. S. C. Black, and N. Kumar, Synthesis, reactivity and biological activity of benzimidazoles, in Bioactive Heterocycles III, Springer, 2007, pp. 87-118, ISBN 978-3-540-73401-7.

11 A. Spasov, I. Yozhitsa, L. Bugaeva and V. Anisimova, Pharm. Chem. J., 1999, 33, 232-243.

12 G. M. Martins, T. Puccinelli, R. A. Gariani, F. R. Xavier, C. C. Silveira and S. R. Mendes, Tetrahedron Lett., 2017, 58, 1969-1972.

13 J. Pogula, S. Laha and P. R. Likhar, Catal. Lett., 2017, 147, 2724-2735.

14 S. Z. K. Hisamoddin, S. Priyanka, S. P. Yogesh and P. Patel Nilam, Pharma Sci. Monit., 2014, 5, 207-225.

15 P. Chaudhary, P. K. Sharma, A. Sharma and J. Varshney, Int. J. Curr. Pharm. Res., 2010, 2, 5-11.

16 I. Hutchinson, M.-S. Chua, H. L. Browne, V. Trapani, T. D. Bradshaw, A. D. Westwell and M. F. Stevens, J. Med. Chem., 2001, 44, 1446-1455.

17 E. Y. Song, N. Kaur, M.-Y. Park, Y. Jin, K. Lee, G. Kim, K. Y. Lee, J. S. Yang, J. H. Shin, K.-Y. Nam, K. T. No and G. Han, Eur. J. Med. Chem., 2008, 43, 1519-1524.

18 P. Vicini, A. Geronikaki, M. Incerti, B. Busonera, G. Poni, C. A. Cabras and P. La Colla, Bioorg. Med. Chem., 2003, 11, 4785-4789.

19 T. Akhtar, S. Hameed, N. Al-Masoudi, R. Loddo and P. La Colla, Acta Pharm., 2008, 58, 135-149.

20 S. Saeed, N. Rashid, P. G. Jones, M. Ali and R. Hussain, Eur. J. Med. Chem., 2010, 45, 1323-1331.

21 F. Russo and M. Santagati, Farmaco Sci., 1976, 31, 41-48.

22 D. Munirajasekhar, M. Himaja, S. V. Mali, A. Karigar and M. Sikarwar, J. Pharma. Res., 2011, 4, 2186-2187.
23 G. Mariappan, P. Prabhat, L. Sutharson, J. Banerjee, U. Patangia and S. Nath, J. Korean Chem. Soc., 2012, 56, 251-256.

24 J. S. Bindra and S. B. Kadin, US Pat. 4,041,163, 1977; A. R. R. Covington, D. L. Temple Jr and J. P. Yevich, US Pat. 4,491,587, 1985.

25 S. Shafi, M. M. Alam, N. Mulakayala, C. Mulakayala, G. Vanaja, A. M. Kalle, R. Pallu and M. Alam, Eur. J. Med. Chem., 2012, 49, 324-333.

26 Y. Liao, H. Qi, S. Chen, P. Jiang, W. Zhou and G.-J. Deng, Org. Lett., 2012, 14, 6004-6007.

27 Z. Zhou and W. Yang, Synth. Commun., 2014, 44, 3189-3198; Y.-S. Lee, Y.-H. Cho, S. Lee, J.-K. Bin, J. Yang, G. Chae and C.-H. Cheon, Tetrahedron, 2015, 71, 532; C. M. Alder, J. D. Hayler, R. K. Henderson, A. M. Redman, L. Shukla, L. E. Shuster and H. F. Sneddon, Green Chem., 2016, 18, 3879-3890.

28 A. J. H. Musser, R. E. Brown, B. Loev, K. Bailey, H. Jones, R. Kahen, F. Huang, A. Khandwala and M. Leibowitz, J. Med. Chem., 1984, 27, 121-125.

29 C. Lau, C. Dufresne, Y. Gareau, R. Zamboni, M. Labelle, R. Young, K. Metters, C. Rochette, N. Sawyer, D. Slipetz, L. Charette, T. Jones, M. McAuliffe, C. McFarlane and A. W. Ford-Hutchinson, Bioorg. Med. Chem. Lett., 1995, 5, 1615-1620.

30 G. Henriksen, A. I. Hauser, A. D. Westwell, B. H. Yousefi, M. Schwaiger, A. Drzezga and H.-J. Wester, J. Med. Chem., 2007, 50, 1087-1089.

31 S. Léger, W. C. Black, D. Deschenes, S. Dolman, J.-P. Falgueyret, M. Gagnon, S. Guiral, Z. Huang, J. Guay, Y. Leblanc, C. S. Li, F. Massé, R. Oballa and L. Zhang, Bioorg. Med. Chem. Lett., 2010, 20, 499-502.

32 B. R. Branchini, M. M. Hayward, S. Bamford, P. M. Brennan and E. J. Lajiness, Photochem. Photobiol., 1989, 49, 689-695.

33 K. Bahrami, M. M. Khodaei and A. Nejati, Green Chem., 2010, 12, 1237-1241.

34 M. A. Chari, D. Shobha and T. Sasaki, Tetrahedron Lett., 2011, 52, 5575-5580.

35 M. Mogharabi-Manzari, M. Kiani, S. Aryanejad, S. Imanparast, M. Amini and M. A. Faramarzi, Adv. Synth. Catal., 2018, 360, 3563-3571.

36 L. Fan, L. Kong and W. Chen, Heterocycles, 2015, 91, 23062314.

37 C. Eggert, U. Temp, J. F. Dean and K.-E. L. Eriksson, FEBS Lett., 1995, 376, 202-206.

38 S. Sayyahi, S. Shabani, S. Ghasemi, A. Azin and S. M. Hasani, Orient. J. Chem., 2015, 31, 1773-1778.

39 Y. Gao, Q. Song, G. Cheng and X. Cui, Org. Biomol. Chem., 2014, 12, 1044-1047.

40 L. S. Gadekar, B. R. Arbad and M. K. Lande, Chin. Chem. Lett., 2010, 21, 1053-1056.

41 L. N. Pushkina, S. A. Mazalov and I. Y. Poatovskii, Zh. Obshch. Khim., 1962, 32, 2624-2633.

42 G. M. Martins, T. Puccinelli, R. A. Gariani, F. R. Xavier, C. C. Silveira and S. R. Mendes, Tetrahedron Lett., 2017, 58, 1969-1972.

43 M. N. Ibrahim, Asian J. Chem., 2007, 19, 2419-2422. 
44 N. D. Kokare, J. N. Sangshetti and D. B. Shinde, Synthesis, 2007, 18, 2829-2834.

45 P. Ghosh and A. Mandal, Catal. Commun., 2011, 12, 744-747.

46 T. G. Deligeorgiev, S. Kaloyanova, A. Vasilev and J. J. Vaquero, Phosphorus, Sulfur Silicon Relat. Elem., 2010, 185, 2292-2302.

47 S. V. Ryabukhin, A. S. Plaskon, D. M. Volochnyuk and A. A. Tolmachev, Synthesis, 2006, 21, 3715-3726.

48 A. K. Chakraborti, S. Rudrawar, G. Kaur and L. Sharma, Synlett, 2004, 1533-1536.
49 Y. M. Ha, J. Y. Park, Y. J. Park, D. Park, Y. J. Choi, J. M. Kim, E. K. Lee, Y. K. Han, J.-A. Kim, J. Y. Lee, H. R. Moon and H. Y. Chung, Bioorg. Med. Chem. Lett., 2011, 21, 2445-2449.

50 Y.-X. Chen, L.-F. Qian, W. Zhang and B. Han, Angew. Chem., Int. Ed., 2008, 47, 9330-9333.

51 H. D. Porter, J. Am. Chem. Soc., 1954, 76, 127-128.

52 M. T. Bogert and A. Stull, J. Am. Chem. Soc., 1926, 48, 248254.

53 M. T. Bogert and H. B. Corbitt, J. Am. Chem. Soc., 1926, 48, 783-788.

54 X. Yang, Y. Xu, J. Chen, J. Ding, H. Wu and W. Su, J. Chem. Res., 2009, 11, 682. 